\title{
Dimension functions, scaling sequences, and wavelet sets
}

\author{
by
}

\author{
Ljiljana Arambašić, Damir Bakić and Rajna Rajić (Zagreb)
}

\begin{abstract}
The paper is a continuation of our study of dimension functions of orthonormal wavelets on the real line with dyadic dilations. The main result of Section 2 is Theorem 2.8 which provides an explicit reconstruction of the underlying generalized multiresolution analysis for any MSF wavelet. In Section 3 we reobtain a result of Bownik, Rzeszotnik and Speegle which states that for each dimension function $D$ there exists an MSF wavelet whose dimension function coincides with $D$. Our method provides a completely new explicit construction of an admissible generalized multiresolution analysis (and, a posteriori, of a wavelet) from an arbitrary dimension function. Several examples are included.
\end{abstract}

1. Introduction. An orthonormal wavelet with dyadic dilations on the real line is a function $\psi \in L^{2}(\mathbb{R})$ for which the system $\left\{\psi_{j, k}(\cdot)=\right.$ $\left.2^{j / 2} \psi\left(2^{j} \cdot-k\right): j, k \in \mathbb{Z}\right\}$ is an orthonormal basis for $L^{2}(\mathbb{R})$. For simplicity, throughout the paper such functions will be called wavelets. An MSF wavelet is a wavelet $\psi$ whose Fourier transform is of the form $\hat{\psi}=\chi_{W}$, where $W$ is a measurable subset of $\mathbb{R}$. Such sets $W$ are called wavelet sets. There are many papers in the literature devoted to various aspects of wavelet sets: their properties, interrelations with other relevant objects, construction methods, etc. We refer the reader to [2], [6], [13], [14, [18] and references therein.

In particular, in [6] all wavelet sets are described in terms of scaling sets. Recall that a measurable set $S \subset \mathbb{R}$ is called a scaling set if $W=2 S \backslash S$ is a wavelet set. In fact, this defines a bijective correspondence: if $W$ is a wavelet set, the corresponding scaling set $S$ is given by $S=\bigcup_{j=1}^{\infty} 2^{-j} W$. It is well known that a measurable set $S \subset \mathbb{R}$ is a scaling set if and only if the following three conditions are satisfied: (i) $2 S \backslash S$ is a $\mathbb{Z}$-tiling domain, (ii) $\frac{1}{2} S \subset S$, up to a null-set, (iii) for a.e. $\xi \in \mathbb{R}$ there exists an integer $j \geq 0$ such that $2^{-j} \xi \in S$. Theorem 2.2 from [6] shows that finding all scaling sets (and hence, a posteriori, all wavelet sets) reduces to finding all measurable

2010 Mathematics Subject Classification: 42C15, 42C40.

Key words and phrases: dimension function, wavelet. 
sets $E \subset \mathbb{R}$ with the property $\frac{1}{2} E \subset E$ together with the associated pairs of so called complementary maps for $E$.

Another interesting method for construction of wavelet sets is presented in [12. Recall that the dimension function $D_{\psi}$ of an orthonormal wavelet $\psi \in L^{2}(\mathbb{R})$ is defined as

$$
D_{\psi}(\xi)=\sum_{j=1}^{\infty} \sum_{k \in \mathbb{Z}}\left|\hat{\psi}\left(2^{j}(\xi+k)\right)\right|^{2}, \quad \xi \in \mathbb{R} .
$$

It is known that $D_{\psi}$ is a well-defined measurable function, non-negative and integer-valued ([3], [15], [19]). An abstract characterization of dimension functions of wavelets is given in Theorem 4.2 of [12]. Moreover, by the same theorem, for each dimension function $D$ there exists an MSF wavelet $\psi$ whose dimension function $D_{\psi}$ coincides with $D$. In fact, the proof of that result provides an algorithm for construction of such a wavelet (i.e., the corresponding wavelet set).

One of the goals of the present article is to give another method for construction of MSF wavelets from dimension functions. This is a continuation of our preceding paper [1. On the technical level, our method uses some of the results obtained in [1. On the other hand, we have developed in [1] a method for construction of dimension functions. In this sense, the results of Section 3 of the present paper describe the second and final step of a new method for construction of MSF wavelets.

In contrast to [12, our approach is based on the multiresolution technique. In a nutshell, our method consists in constructing a suitable generalized multiresolution analysis (GMRA; see Section 2 for relevant definitions and facts). In this way, similarly to [6], we focus our attention on scaling sets, rather than wavelet sets. The difference is that our construction provides not only a scaling set, but also the scaling sequence of the underlying multiresolution structure. Since the number of generators (i.e., scaling functions) is equal to the essential maximum of the dimension function, only MRA wavelets can arise from a single scaling function. In all other cases, a sequence (possibly finite) of scaling functions is needed. This means that most scaling sets have a complex structure. Our construction gives an insight into this structure by describing a scaling set in terms of its constituting components.

It is known that each wavelet arises from a uniquely determined GMRA. Thus, the corresponding GMRA serves not only as a tool, but also provides some intrinsic information. Moreover, there is some evidence that a precise description of an MSF wavelet in terms of the underlying generalized multiresolution structure may lead to a technique for building more sophisticated wavelets from MSF ones. For a more specific comment on this we refer to Remark 2.11 and Examples 2.12, 2.13 below. 
We point out that both aforementioned papers which are concerned with wavelet sets, 6] and [12, are written in a general setting of dilations in $L^{2}\left(\mathbb{R}^{n}\right)$ induced by expanding matrices with integer coefficients. In the present paper we restrict ourselves to the dyadic case on the real line. The main reason for doing so is that we need some auxiliary results that are available in the literature only in the dyadic case, and extending such results to $n$ dimensions, even when straightforward, would make our exposition unreasonably long. In particular, this applies to the results from [7] that serve as the key technical tool in Section 2.

The paper is organized as follows. In Section 2 we summarize necessary technical results on GMRA's from the literature. Proposition 2.7 gives a simple formula for a wavelet arising from a GMRA with the scaling system consisting of characteristic functions. Theorem 2.8 provides an explicit description of the underlying GMRA for any given MSF wavelet. At the end of the section we describe a possible application of Theorem 2.8 a scheme that can be used to construct new wavelets from MSF ones. As an illustration of the proposed technique, we construct two examples of non-MSF wavelets.

In Section 3 we describe our method for construction of wavelets from dimension functions. It is well known that many different wavelets may share the same dimension function (notice that the dimension function of each MRA wavelet is almost identically equal to 1 ). Given a dimension function $D$, we construct an MSF wavelet $\psi$ with $D_{\psi}=D$. In fact, our method provides an explicit GMRA such that the dimension function (i.e., the multiplicity function) of its core space coincides with $D$. The section ends with a couple of examples which illustrate our technique.

Throughout the paper we use the Fourier transform in the form $\hat{f}(\xi)=$ $\int_{\mathbb{R}} f(x) e^{-2 \pi i x \xi} d x$.

By $|S|$ we denote the Lebesgue measure of a set $S \subseteq \mathbb{R}$.

The space of square integrable functions on the 1-dimensional torus $\mathbb{T}=$ $\mathbb{R} / \mathbb{Z}$ is denoted by $L^{2}(\mathbb{T})$.

We denote by $\tau: \mathbb{R} \rightarrow[-1 / 2,1 / 2)$ the translation projection onto the unit interval. We say that measurable sets $S, T \subseteq \mathbb{R}$ are congruent and write $S \equiv T$ if there exists a measurable partition $\left(S_{k}\right), k \in \mathbb{Z}$, of $S$ such that $\left(S_{k}+k\right), k \in \mathbb{Z}$, is, up to a null-set, a partition of $T$.

2. Admissible GMRA's revisited. Our goal in this section is to describe the interrelation between an MSF wavelet and the underlying multiresolution structure. It is known how to obtain wavelets associated with a given generalized multiresolution analysis (see Remark 2.6 below). A related result in the opposite direction, namely, an explicit reconstruction of the associated generalized multiresolution analysis from a given MSF wavelet (Theorem 2.8) is new. 
Let us begin by summarizing some relevant definitions and facts well known from the literature.

Definition 2.1. A sequence $\left(V_{j}\right), j \in \mathbb{Z}$, of closed subspaces of $L^{2}(\mathbb{R})$ is said to be a generalized multiresolution analysis (GMRA) if the following conditions are satisfied:

(1) $V_{j} \subseteq V_{j+1}$ for all $j$;

(2) $D V_{j}=V_{j+1}$ for all $j$ (where $D f(x)=\sqrt{2} f(2 x), f \in L^{2}(\mathbb{R})$ );

(3) $\bigcap_{j} V_{j}=\{0\}, \overline{\bigcup_{j} V_{j}}=L^{2}(\mathbb{R})$;

(4) the core space $V_{0}$ is a shift invariant space (i.e., $T_{k} V_{0} \subseteq V_{0}$ for all $k \in \mathbb{Z}$, where $\left.T_{k} f(x)=f(x-k), f \in L^{2}(\mathbb{R})\right)$.

Recall that each shift invariant space $V_{0}$ admits a standard decomposition of the form $V_{0}=\bigoplus_{i=1}^{\infty}\left\langle\varphi_{i}\right\rangle$, where $\left\langle\varphi_{i}\right\rangle$ is the minimal closed shift invariant subspace of $L^{2}(\mathbb{R})$ that contains $\varphi_{i}$ and, additionally, the system $\left\{T_{k} \varphi_{i}\right.$ : $k \in \mathbb{Z}\}$ is a Parseval frame for $\left\langle\varphi_{i}\right\rangle$ for all $i \in \mathbb{N}$. Since $\varphi_{i}$ is a Parseval generator for $\left\langle\varphi_{i}\right\rangle$, there exists a measurable $\mathbb{Z}$-periodic set $\Omega_{i} \subseteq \mathbb{R}$ such that $\sigma_{\varphi_{i}}(\xi):=\sum_{k \in \mathbb{Z}}\left|\hat{\varphi}_{i}(\xi+k)\right|^{2}=\chi_{\Omega_{i}}(\xi)$ a.e. In addition, one can choose the generators $\varphi_{i}$ (which are called scaling functions when $V_{0}$ is the core space of some GMRA) of $V_{0}$ so that $\Omega_{1} \supseteq \Omega_{2} \supseteq \cdots$. The function $\xi \mapsto \sum_{i=1}^{\infty} \chi_{\Omega_{i}}(\xi)$ is called the multiplicity function (or the dimension function) of $V_{0}$.

It may happen that $V_{0}$ has only finitely many, say $n$, scaling functions. If this is the case, we shall still write $V_{0}=\bigoplus_{i=1}^{\infty}\left\langle\varphi_{i}\right\rangle$ assuming that $\varphi_{i}=0$ and $\Omega_{i}=\emptyset$ for all $i>n$.

We shall frequently work with scaling functions $\varphi_{i}, i \in \mathbb{N}$, such that $\hat{\varphi}_{i}=\chi_{S_{i}}$, where each $S_{i}$ is a measurable set. A description of such sets is obtained in Proposition 4.1 of [7]. For the reader's convenience we restate that description in the following remark.

REMARK 2.2. Let $\left(S_{i}\right)$ be a finite or infinite sequence of measurable subsets of $\mathbb{R}$. Suppose that $\Omega_{1} \supseteq \Omega_{2} \supseteq \cdots$, where $\Omega_{i}=S_{i}+\mathbb{Z}, i \in \mathbb{N}$. Let $\hat{\varphi}_{i}=\chi_{S_{i}}, i \in \mathbb{N}$. Then $\left(\varphi_{i}\right)$ is a sequence of scaling functions for a GMRA if the following conditions are satisfied:

(1) $\left|S_{i} \cap S_{j}\right|=0, i \neq j$;

(2) $\left|S_{i} \cap\left(S_{i}+k\right)\right|=0, k \in \mathbb{Z} \backslash\{0\}, i \in \mathbb{N}$;

(3) $\frac{1}{2} S_{i} \subseteq \bigcup_{j=1}^{\infty} S_{j}, i \in \mathbb{N}$, up to a null-set;

(4) $\sum_{i=1}^{\infty}\left|S_{i}\right|<\infty$;

(5) for a.e. $\xi$ there exists $m(\xi) \in \mathbb{N} \cup\{0\}$ such that $2^{-m(\xi)} \xi \in \bigcup_{j=1}^{\infty} S_{j}$.

Given a GMRA $\left(V_{j}\right)$, one defines a new sequence of subspaces $\left(W_{j}\right)$, $j \in \mathbb{Z}$, by $W_{j}=V_{j+1} \ominus V_{j}$. It turns out that $W_{0}$ is shift invariant, $W_{j}=D^{j} W_{0}$ for all $j \in \mathbb{Z}$, and $L^{2}(\mathbb{R})=\bigoplus_{j \in \mathbb{Z}} W_{j}$. Hence, if there exists a function 
$\psi \in W_{0}$ such that $W_{0}=\langle\psi\rangle$ and $\psi$ is an orthonormal generator for $W_{0}$ (i.e., $\left\{T_{k} \psi: k \in \mathbb{Z}\right\}$ is an orthonormal basis for $\left.W_{0}\right)$, then, clearly, $\psi$ is a wavelet.

DeFinition 2.3. We say that a GMRA $\left(V_{j}\right)$ is admissible if $W_{0}$, as a shift invariant space, has a single orthonormal generator $\psi$. We then say that $\psi$ is a wavelet associated with $\left(V_{j}\right)$.

Our interest in GMRA's arises from the following well-known fact:

REMARK 2.4. Each wavelet $\psi$ is associated with a uniquely determined GMRA.

Indeed, let $V_{0}=\overline{\operatorname{span}}\left\{D^{j} T_{k} \psi: j<0, k \in \mathbb{Z}\right\}$ and, for $j \in \mathbb{Z}, V_{j}=D^{j} V_{0}$. It turns out that $\left(V_{j}\right)$ is a GMRA for which $W_{0}=V_{1} \ominus V_{0}=\langle\psi\rangle$; in other words, $\left(V_{j}\right)$ is an admissible GMRA and $\psi$ is a wavelet associated to it.

If $\left(V_{j}^{\prime}\right)$ is any GMRA such that $W_{0}^{\prime}:=V_{1}^{\prime} \ominus V_{0}^{\prime}=\langle\psi\rangle$, one easily concludes that $V_{0}^{\prime}=\overline{\operatorname{span}}\left\{D^{j} T_{k} \psi: j<0, k \in \mathbb{Z}\right\}=V_{0}$.

By the preceding remark, all wavelets arise from admissible GMRA's. Thus, it is important to recognize admissible ones among all GMRA's. For the reader's convenience we shall restate a relevant result (keeping the notations of the discussion following Definition 2.1) in our next remark. For the details we refer the reader to [6], [10] and [8].

REMARK 2.5. A GMRA $\left(V_{j}\right)$ is admissible if and only if the following two conditions are satisfied:

(1) $\sum_{i=1}^{\infty} \chi_{\Omega_{i}}(\xi)<\infty$ a.e. (i.e., $\left|\bigcap_{i=1}^{\infty} \Omega_{i}\right|=0$ );

(2) $\sum_{i=1}^{\infty} \chi_{\Omega_{i}}(\xi)+\sum_{i=1}^{\infty} \chi_{\Omega_{i}}(\xi+1 / 2)-\sum_{i=1}^{\infty} \chi_{\Omega_{i}}(2 \xi)=1$ a.e.

If $\left(V_{j}\right)$ is an admissible GMRA and if $\psi$ is an associated wavelet then it is also known that $D_{\psi}(\xi)=\sum_{i=1}^{\infty} \chi_{\Omega_{i}}(\xi)$ a.e.

Notice that the first condition is trivially fulfilled when $V_{0}$ is finitely generated as a shift invariant space.

We conclude this introductory part by presenting a method for obtaining wavelets associated with a given admissible GMRA. The following remark summarizes several results from [7]. A similar technique is described in [11] in terms of so called low-pass and high-pass matrix masks; see a generalized Unitary Extension Principle in Theorem 3.1 of [11].

REMARK 2.6. Let $\left(V_{j}\right)$ be an admissible GMRA, let $V_{0}=\bigoplus_{i=1}^{\infty}\left\langle\varphi_{i}\right\rangle$, $\sigma_{\varphi_{i}}(\xi)=\chi_{\Omega_{i}}(\xi)$ a.e., $\Omega_{1} \supseteq \Omega_{2} \supseteq \cdots$. Let us describe all wavelets associated with $\left(V_{j}\right)$ in terms of the sequence $\left(\varphi_{i}\right)$ of scaling functions. It suffices to obtain one associated wavelet $\psi$, since then, by the general theory of shift invariant spaces, any other associated wavelet $\psi_{s}$ is given by $\hat{\psi}_{s}(\xi)=s(\xi) \hat{\psi}(\xi)$ where $s$ is some measurable, a.e. unimodular, and $\mathbb{Z}$-periodic function. 
First, for each $f \in V_{1}$, there exists a unique sequence $\mu(f)=t=\left(t_{j}\right)$ of functions $t_{j} \in L^{2}(\mathbb{T}), j \in \mathbb{N}$, such that $\hat{f}(2 \xi)=\sum_{j=1}^{\infty} t_{j}(\xi) \hat{\varphi}_{j}(\xi)$ a.e. and $t_{j}(\xi)=0$ for all $\xi \notin \Omega_{j}$ and each $j$. Notice that the sum is finite when $V_{0}$ is finitely generated. When $V_{0}$ has infinitely many generators, the above series converges in norm. However, by condition (1) of Remark 2.5, for a.e. $\xi$ there exists a natural number $j(\xi)$ such that $\xi \notin \Omega_{j}$ for $j>j(\xi)$. Thus, the above sum is finite for a.e. $\xi$.

Let $\mu\left(\varphi_{i}\right)=m_{i}=\left(m_{i j}\right), i \in \mathbb{N}$. The sequence $m_{i}=\left(m_{i j}\right)$ is called the minimal filter for the scaling function $\varphi_{i}$ and satisfies

$$
\begin{aligned}
& \hat{\varphi}_{i}(2 \xi)=\sum_{j=1}^{\infty} m_{i j}(\xi) \hat{\varphi}_{j}(\xi) \quad \text { a.e. }, \\
& m_{i j}(\xi)=0, \quad \forall \xi \notin \Omega_{j}, \forall j \in \mathbb{N} .
\end{aligned}
$$

If we define, for each $i \in \mathbb{N}$ and $\xi \in \mathbb{R}$, a double sequence

$$
m_{i}^{d}(\xi)=\left(m_{i 1}(\xi), m_{i 2}(\xi), \ldots, m_{i 1}(\xi+1 / 2), m_{i 2}(\xi+1 / 2), \ldots\right),
$$

then $m_{i}^{d}(\xi) \perp m_{k}^{d}(\xi)$ for $i \neq k$ and a.e. $\xi$ (in the sense $\sum_{j=1}^{\infty} m_{i j}(\xi) \overline{m_{k j}(\xi)}+$ $\left.\sum_{j=1}^{\infty} m_{i j}(\xi+1 / 2) \overline{m_{k j}(\xi+1 / 2)}=0\right)$ and $\sum_{j=1}^{\infty}\left|m_{i j}(\xi)\right|^{2}+\sum_{j=1}^{\infty} \mid m_{i j}(\xi+$ $1 / 2)\left.\right|^{2}=\left\|m_{i}^{d}(\xi)\right\|^{2}=\chi_{\Omega_{i}}(2 \xi)$ for each $i$ and a.e. $\xi$.

By Propositions 2.7 and 2.9 of [7], there exists a sequence $v=\left(v_{j}\right)$ of functions $v_{j} \in L^{2}(\mathbb{T})$ satisfying $v_{j}(\xi)=0$ for all $\xi \notin \Omega_{j}$ and all $j$, such that the sequence $v^{d}(\xi)=\left(v_{1}(\xi), v_{2}(\xi), \ldots, v_{1}(\xi+1 / 2), v_{2}(\xi+1 / 2), \ldots\right), \xi \in \mathbb{R}$, has the properties:

(1) $v^{d}(\xi) \perp m_{i}^{d}(\xi)$ for each $i$ and a.e. $\xi$;

(2) $\left\|v^{d}(\xi)\right\|^{2}=1$ a.e.

A sequence $\left(v_{j}\right)$ with these properties is uniquely determined if we additionally require that the first non-trivial component of $v^{d}(\xi)$ is positive, for a.e. $\xi$.

Finally, by Theorem 1.9 of [7], if $\left(v_{j}\right)$ is a sequence of functions in $L^{2}(\mathbb{T})$ satisfying $v_{j}(\xi)=0$ for all $\xi \notin \Omega_{j}$ and all $j$, and the above conditions (1) and (2), then the function $\psi$ defined by $\hat{\psi}(2 \xi)=\sum_{j=1}^{\infty} v_{j}(\xi) \hat{\varphi}_{j}(\xi)$ is a wavelet associated with $\left(V_{j}\right)$.

Our first proposition is known and we include it only for completeness. It can be proved using the technique of the preceding remark, but here we include a sketch of a more direct proof as proposed by the referee.

Proposition 2.7. Let $\left(V_{j}\right)$ be an admissible GMRA such that $V_{0}=$ $\bigoplus_{i=1}^{\infty}\left\langle\varphi_{i}\right\rangle, \hat{\varphi}_{i}=\chi_{S_{i}}, i \in \mathbb{N}$. Then an associated wavelet $\psi$ is given by $\hat{\psi}=\chi_{W}$ where $W=2 S \backslash S$ and $S=\bigcup_{i=1}^{\infty} S_{i}$. 
Proof. Denoting by ${ }^{-}$the inverse Fourier transform, we have $V_{0}=\check{L}^{2}(S)$ and hence $V_{1}=\check{L}^{2}(2 S)$. Thus, $W_{0}=V_{1} \ominus V_{0}=\check{L}^{2}(W)$ where $W=2 S \backslash S$. So, $\hat{\psi}=\chi_{W}$ must be a wavelet associated with $\left(V_{j}\right)$.

Suppose now that we are given an MSF wavelet $\psi, \hat{\psi}=\chi_{W}$. By Remark 2.4 $\psi$ is associated with a unique GMRA $\left(V_{j}\right)$. Now we want to reconstruct the core space $V_{0}$, in other words, the sequence of scaling functions $\left(\varphi_{i}\right)$.

Recall that $S=\bigcup_{j=1}^{\infty} 2^{-j} W$ is the corresponding scaling set.

For $\xi \in \tau(S)$ define $m_{1}(\xi) \in \mathbb{Z}$ by

$$
\left|\xi+m_{1}(\xi)\right|=\min \{|\xi+m|: m \in \mathbb{Z}, \xi+m \in S\}
$$

and

$$
M_{1}=\left\{\xi+m_{1}(\xi): \xi \in \tau(S)\right\} .
$$

Notice that $\left|\xi+m_{1}\right|=\left|\xi+m_{2}\right|$ with $\xi \in[-1 / 2,1 / 2)$ and $m_{1}, m_{2} \in \mathbb{Z}$, $m_{1} \neq m_{2}$, implies $\xi=-1 / 2$ or $\xi=0$. This shows that the function $m_{1}$ is well defined on the set $\tau(S) \backslash\{-1 / 2,0\}$. It is also easy to see that $M_{1}$ is a measurable set.

By defining $M_{1}$ we have chosen a unique representative (the smallest in absolute value) in the congruence class for each point in $S \backslash \frac{1}{2} \mathbb{Z}$. Let us proceed by induction.

Suppose we have pairwise disjoint, measurable sets $M_{1}, \ldots, M_{n} \subseteq S$ such that the restriction of the translation projection $\tau$ to each $M_{i}$ is an injection. For $\xi \in \tau\left(S \backslash \bigcup_{i=1}^{n} M_{i}\right), \xi \notin\{-1 / 2,0\}$, define $m_{n+1}(\xi)$ by

$$
\left|\xi+m_{n+1}(\xi)\right|=\min \left\{|\xi+m|: m \in \mathbb{Z}, \xi+m \in S \backslash \bigcup_{i=1}^{n} M_{i}\right\}
$$

and

$$
M_{n+1}=\left\{\xi+m_{n+1}(\xi): \xi \in \tau\left(S \backslash \bigcup_{i=1}^{n} M_{i}\right)\right\} .
$$

Clearly, this gives us a sequence (possibly finite) of pairwise disjoint measurable sets $\left(M_{i}\right)$ such that $S \backslash \frac{1}{2} \mathbb{Z}=\bigcup_{i=1}^{\infty} M_{i}$. Thus, up to a set of measure zero, the sequence $\left(M_{i}\right)$ makes up a partition of $S$. For $i \in \mathbb{N}$, define $\varphi_{i} \in L^{2}(\mathbb{R})$ by $\hat{\varphi}_{i}=\chi_{M_{i}}$ and $\Omega_{i}=M_{i}+\mathbb{Z}$. It follows from the construction that the sequence $\left(\Omega_{i}\right)$ decreases.

We claim that $\left(\varphi_{i}\right)$ is a sequence of scaling functions for a GMRA $\left(V_{j}\right)$ whose core space is $V_{0}=\bigoplus_{i=1}^{\infty}\left\langle\varphi_{i}\right\rangle$.

To see this, we need to check the conditions of Remark 2.2. Conditions (1) and (2) follow immediately from the construction, while (4) is trivially satisfied since $S$, as a scaling set, has Lebesgue measure equal to 1 . The 
remaining conditions (3) and (5) are direct consequences of conditions (ii) and (iii) in Theorem 3.3 of [12].

It remains to show that $\left(V_{j}\right)$ is the underlying GMRA for $\psi$. First observe that $D_{\psi}(\xi)=\sum_{j=1}^{\infty} \sum_{k \in \mathbb{Z}}\left|\hat{\psi}\left(2^{j}(\xi+k)\right)\right|^{2}=\sum_{j=1}^{\infty} \sum_{k \in \mathbb{Z}} \chi_{W}\left(2^{j}(\xi+k)\right)=$ $\sum_{j=1}^{\infty} \sum_{k \in \mathbb{Z}} \chi_{2^{-j} W}(\xi+k)=\sum_{k \in \mathbb{Z}} \chi_{S}(\xi+k)=\sum_{i=1}^{\infty} \chi_{\Omega_{i}}(\xi)$. This, together with Theorem 4.2 of [12, directly implies properties (1) and (2) of Remark 2.5. hence, $\left(V_{j}\right)$ is an admissible GMRA.

We are now in a position to apply the preceding proposition: an associated wavelet is an MSF wavelet whose wavelet set is $2 S \backslash S$; since $2 S \backslash S=W$, this is precisely our original wavelet $\psi$.

This completes the proof of the following theorem.

TheOREm 2.8. Let $\psi$ be an MSF wavelet such that $\hat{\psi}=\chi_{W}$. Define a sequence $\left(\varphi_{i}\right)$ of functions $\varphi_{i} \in L^{2}(\mathbb{R})$ by $\hat{\varphi}_{i}=\chi_{M_{i}}, i \in \mathbb{N}$, where the sets $M_{i}$ are given by (2.1) and (2.2). Then $\psi$ arises from the $G M R A\left(V_{j}\right)$ with core space $V_{0}=\bigoplus_{i=1}^{\infty}\left\langle\varphi_{i}\right\rangle$.

Example 2.9. Consider the Journé wavelet set $W=[-16 / 7,-2) \cup$ $[-1 / 2,-2 / 7) \cup[2 / 7,1 / 2) \cup[2,16 / 7)$. The corresponding scaling set is $S=$ $[-8 / 7,-1) \cup[-4 / 7,-1 / 2) \cup[-2 / 7,2 / 7) \cup[1 / 2,4 / 7) \cup[1,8 / 7)$. Following the construction from the above proof, one obtains $M_{1}=[-4 / 7,-1 / 2) \cup$ $[-2 / 7,2 / 7) \cup[1 / 2,4 / 7)$ and $M_{2}=[-8 / 7,-1) \cup[1,8 / 7)$.

REMARK 2.10. The scaling sequence $\left(\varphi_{i}\right)$ with $\hat{\varphi}_{i}=\chi_{M_{i}}$, obtained in the preceding theorem, is not unique. To see this, take any $n \geq 2$ and an arbitrary measurable set $E \subseteq M_{n}$. Let $k<n$. Since $\Omega_{n}=M_{n}+\mathbb{Z} \subseteq \Omega_{k}=M_{k}+\mathbb{Z}$, there is a measurable set $F \subseteq M_{k}$ such that $F \equiv E$. It is easy to see that the sequence $\left(S_{i}\right)$ defined by $S_{n}=\left(M_{n} \backslash E\right) \cup F, S_{k}=\left(M_{k} \backslash F\right) \cup E, S_{i}=M_{i}$ for $i \neq n, k$, satisfies the conditions of Remark 2.2 hence, the sequence $\left(\phi_{i}\right)$ defined by $\hat{\phi}_{i}=\chi_{S_{i}}$ is a new scaling sequence. Since $S_{i}+\mathbb{Z}=M_{i}+\mathbb{Z}=\Omega_{i}$ for all $i$, and $\bigcup_{i=1}^{\infty} S_{i}=\bigcup_{i=1}^{\infty} M_{i}=S$, the resulting GMRA produces the same wavelet $\psi$. Recalling that each wavelet has a uniquely determined GMRA, we conclude that the sequences $\left(M_{i}\right)$ and $\left(S_{i}\right)$ generate the same GMRA. In fact, we have obtained two different standard decompositions of the core space: $V_{0}=\bigoplus_{i=1}^{\infty}\left\langle\varphi_{i}\right\rangle=\bigoplus_{i=1}^{\infty}\left\langle\phi_{i}\right\rangle$.

As is known from the literature, there are several techniques for construction of wavelet sets (i.e., MSF wavelets). The class of MSF wavelets is an important source of examples and counterexamples. However, MSF wavelets are not very useful in applications due to discontinuities of their Fourier transforms. Hence, it is natural to ask: is it possible to build new wavelets from MSF ones? Theorem 2.8 may be used as a first step toward achieving that goal. 
REMARK 2.11. Given an MSF wavelet $\psi$ one can explore the following scheme. First, by using Theorem 2.8, one reconstructs the underlying GMRA, i.e., the sequence $\left(M_{i}\right)$. Put $\Omega_{i}=M_{i}+\mathbb{Z}, i \in \mathbb{N}$. By Remark 2.5, we know that the sequence $\left(\Omega_{i}\right)$ satisfies admissibility conditions (1) and (2) of Remark 2.5. The idea is now to find a new sequence of scaling functions $\left(\phi_{i}\right)$ for another GMRA $\left(U_{j}\right)$ such that $\left\{\xi \in \mathbb{R}: \hat{\phi}_{i}(\xi) \neq 0\right\}+\mathbb{Z}=\Omega_{i}, i \in \mathbb{N}$. To be more specific, we want to find sequences $\left(P_{i}\right)$ and $\left(R_{i}\right)$ of measurable sets such that $P_{i} \subseteq M_{i}$ and $P_{i}+\mathbb{Z}=R_{i}+\mathbb{Z}, i \in \mathbb{N}$, and a sequence of functions $\left(\phi_{i}\right)$ such that $\left\{\xi \in \mathbb{R}: \hat{\phi}_{i}(\xi) \neq 0\right\}=M_{i} \cup R_{i}, i \in \mathbb{N}$. If this is done in such a way that $\left(\phi_{i}\right)$ is a scaling sequence, then the core space $U_{0}$ of the resulting GMRA $\left(U_{j}\right)$ has the same multiplicity function, since $\sigma_{\phi_{i}}=\sigma_{\varphi_{i}}=\chi_{\Omega_{i}}$ for all $i \in \mathbb{N}$. Hence, $\left(U_{j}\right)$ is a new admissible GMRA. To obtain associated wavelets, it only remains to apply the technique described in Remark 2.6.

In the following two examples we illustrate the proposed method.

ExAmple 2.12. Let $W=[-2 / 3,-1 / 3) \cup[2 / 3,4 / 3)$. Since each of the families $(W+k), k \in \mathbb{Z}$, and $\left(2^{j} W\right), j \in \mathbb{Z}$, makes up a partition of $\mathbb{R}, W$ is a wavelet set, i.e., the function $\psi$ defined by $\hat{\psi}=\chi_{W}$ is an MSF wavelet. It is easy to see that $D_{\psi} \equiv 1$; thus, $\psi$ is an MRA wavelet. The corresponding scaling set is $S=\bigcup_{j=1}^{\infty} 2^{-j} W=[-1 / 3,2 / 3)$. As in the proof of Theorem 2.8 . one finds $M_{1}=S$ and $M_{n}=\emptyset$ for all $n \geq 2$. This shows that $\psi$ arises from an MRA $\left(V_{j}\right)$, where $V_{0}=\langle\varphi\rangle$ and $\hat{\varphi}=\chi_{S}$. In particular, if we denote $\Omega=S+\mathbb{Z}$, we know that $\chi_{\Omega}(\xi)+\chi_{\Omega}(\xi+1 / 2)-\chi_{\Omega}(2 \xi)=1$ for all $\xi \in \mathbb{R}$ (which is obvious in this situation; since $S$ is a $\mathbb{Z}$-tiling domain, $\Omega=\mathbb{R}$ ).

Let $P=[-1 / 3,-1 / 6) \subset S$ and $R=P+1 \equiv P$. Choose arbitrary $L^{2}$-functions $a$ and $b$ on $P$ such that $|a(\xi)|^{2}+|b(\xi)|^{2}=1$ for all $\xi \in P$ and $0<|a(\xi)|<1$ for a.e. $\xi \in P$. Now define a function $\phi$ by

$$
\hat{\phi}(\xi)= \begin{cases}1, & \xi \in S \backslash P, \\ a(\xi), & \xi \in P, \\ b(\xi-1), & \xi \in P+1, \\ 0, & \text { otherwise. }\end{cases}
$$

Notice that $\{\xi \in \mathbb{R}: \hat{\phi}(\xi) \neq 0\}=S \cup R$ and $(S \cup R)+\mathbb{Z}=S+\mathbb{Z}$. One easily finds that $\sigma_{\phi}(\xi)=1$ for all $\xi \in \mathbb{R}$, which shows that, as was the key idea in the preceding discussion, by passing from $\varphi$ to $\phi$ we did not change the original set $\Omega=\mathbb{R}$. Furthermore, an easy application of Theorem 7.5.2 of [17] shows that $\phi$ is a scaling function. Hence, the sequence of subspaces $\left(U_{j}\right), j \in \mathbb{Z}$, where $U_{j}=D^{j} U_{0}$ and $U_{0}=\langle\phi\rangle$, is a new MRA. To obtain associated wavelets, we follow the procedure of Remark 2.6 which, in this situation, reduces to the well known high pass filter technique. After a simple computation (we omit the details) one obtains a wavelet $\psi_{\phi}$ which is given 
by

$$
\widehat{\psi_{\phi}}(2 \xi)= \begin{cases}\frac{a(\xi),}{\overline{b(2 \xi),}} & \xi \in P=[-1 / 3,-1 / 6), \\ \frac{-a(2 \xi-1),}{-} & \xi \in 1 / 2 P=[-1 / 6,-1 / 12), \\ 1, & \xi \in[5 / 12,2 / 3), \\ b(\xi-1), & \xi \in P+1=[2 / 3,5 / 6), \\ 0, & \text { otherwise. }\end{cases}
$$

We shall now specify $a$ and $b$. Our goal is to obtain a wavelet $\psi_{\phi}$ whose Fourier transform $\widehat{\psi_{\phi}}$ is a smooth function. Put

$$
\begin{array}{ll}
a(\xi)=\sin (f(\xi)), & \xi \in[-1 / 3,-1 / 6), \\
b(\xi)=\cos (f(\xi)), \quad \xi \in[-1 / 3,-1 / 6),
\end{array}
$$

where $f$ is a temporarily unspecified real function. Obviously, such $a$ and $b$ satisfy the condition $|a(\xi)|^{2}+|b(\xi)|^{2}=1$ for all $\xi \in P=[-1 / 3,-1 / 6)$.

Finally, observe that if $f$ is a $C^{\infty}$-function defined on some open set that contains $P$ with the properties

$$
f^{(n)}\left(-\frac{1}{3}\right)=f^{(n)}\left(-\frac{1}{6}\right)=0, \quad \forall n \in \mathbb{N}, \quad f\left(-\frac{1}{3}\right)=0, \quad f\left(-\frac{1}{6}\right)=\frac{\pi}{2},
$$

then (2.3) gives us a wavelet $\psi_{\phi, \infty}$ with $C^{\infty}$ Fourier transform. To obtain such an $f$, consider first the function $g$ defined by

$$
g(\xi)= \begin{cases}e^{\frac{1}{(\xi+1 / 6)(\xi+1 / 3)}}, & \xi \in(-1 / 3,-1 / 6), \\ 0, & \text { otherwise. }\end{cases}
$$

An easy computation shows that $g$ is a $C^{\infty}$-function with $g^{(n)}(-1 / 3)=$ $g^{(n)}(-1 / 6)=0$ for all $n \in \mathbb{N} \cup\{0\}$. The only problem is that the value of $g$ at $-1 / 6$ is not $\pi / 2$, but 0 . To adjust $g$ appropriately, let $K=\int_{-1 / 3}^{-1 / 6} g(t) d t$ and

$$
f(\xi)=\frac{\pi}{2 K} \int_{-1 / 3}^{\xi} g(t) d t
$$

Obviously, $f$ is an increasing function with $f(-1 / 3)=0$ and $f(-1 / 6)$ $=\pi / 2$. Moreover, since $f^{(n)}(\xi)=\frac{\pi}{2 K} g^{(n-1)}(\xi)$ for all $n \in \mathbb{N}$, we also have $f^{(n)}(-1 / 3)=f^{(n)}(-1 / 6)=0$ for all $n \in \mathbb{N}$.

We note that a similar family of smooth wavelets is obtained in [16] by a different technique. Example 1 of [16] describes an explicit construction of a smooth wavelet $\psi_{\alpha}$ with $\operatorname{supp}\left|\widehat{\psi_{\alpha}}\right| \subset S_{\alpha}=\left[-\frac{4}{3 \pi} \alpha, 2-\frac{2}{3 \pi} \alpha\right)$ for any $\alpha \in(0, \pi]$. Notice that for $\alpha=\pi / 2$ this gives $S_{\pi / 2}=[-2 / 3,5 / 3)$, which is 


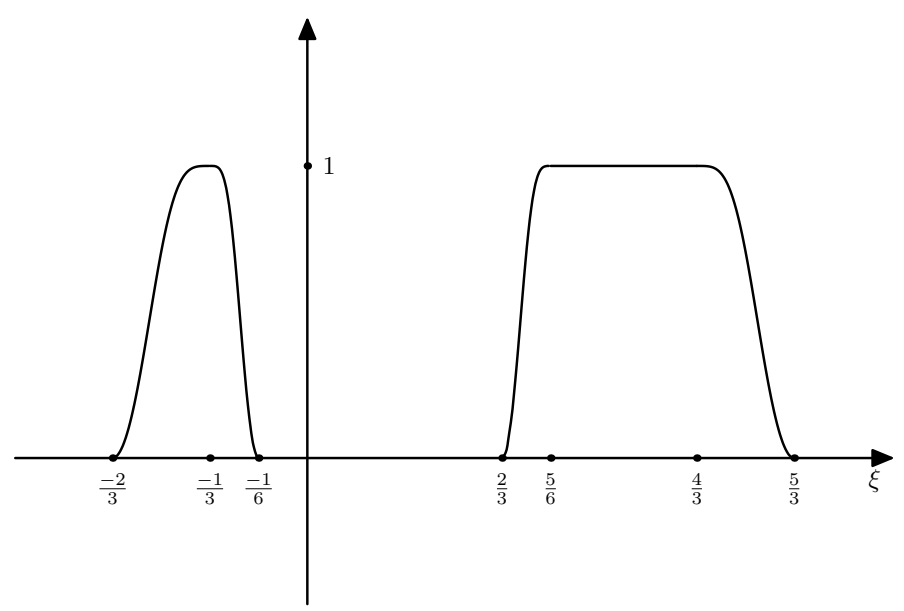

Fig. 1. The graph of $\widehat{\psi_{\phi, \infty}}$

precisely the minimal interval that contains the support of $\widehat{\psi_{\phi, \infty}}$ given by (2.3).

Let us also mention that the technique of Example 2.12 cannot be extended to the class of non-MRA MSF wavelets if we want to obtain a wavelet with a continuous compactly supported Fourier transform. This is a direct consequence of Theorem 3.12 of [16. Thus, if we want to produce a non-MRA wavelet with a continuous Fourier transform by using the technique of Remark 2.11, we must choose sets $R_{i}$ in such a way that the Fourier transform of the resulting wavelet has an unbounded support.

In our next example we construct a non-MSF non-MRA wavelet $\psi$ whose Fourier transform is compactly supported (and hence discontinuities of $\hat{\psi}$ must be present).

Example 2.13. Consider Journé's dimension function

$$
D(\xi)= \begin{cases}2, & \xi \in[-1 / 7,1 / 7)+\mathbb{Z}, \\ 1, & \xi \in([-1 / 2,-3 / 7) \cup[-2 / 7,-1 / 7) \cup[1 / 7,2 / 7) \cup[3 / 7,1 / 2))+\mathbb{Z}, \\ 0, & \text { otherwise, }\end{cases}
$$

and an MSF wavelet $\chi_{W}$ where $W=2 S \backslash S, S=M_{1} \cup M_{2}$ and $M_{1}=[-1 / 2,-3 / 7) \cup[-2 / 7,2 / 7) \cup[3 / 7,1 / 2), \quad M_{2}=[-1,-6 / 7) \cup[6 / 7,1)$. Observe that $D_{\psi}=D$ (cf. Example 3.19).

The corresponding scaling functions are given by $\hat{\varphi}_{1}=\chi_{M_{1}}$ and $\hat{\varphi}_{2}=$ $\chi_{M_{2}}$. Here we introduce new scaling functions $\phi_{1}$ and $\phi_{2}$ in the following way. First, let $a$ and $b$ be measurable functions on $[-4 / 7,-3 / 7)$ such that 


$$
\begin{gathered}
|a(\xi)|^{2}+|b(\xi)|^{2}=1 \text { for all } \xi \in[-4 / 7,-3 / 7) \text {. Now put } \\
\hat{\phi}_{1}(\xi)= \begin{cases}a(\xi), & \xi \in[-4 / 7,-3 / 7), \\
1, & \xi \in[-2 / 7,2 / 7), \\
b(\xi-1), & \xi \in[3 / 7,4 / 7), \\
0, & \text { otherwise, }\end{cases} \\
\hat{\phi}_{2}(\xi)= \begin{cases}a(\xi / 2), & \xi \in[-8 / 7,-6 / 7), \\
b(\xi / 2-1), & \xi \in[6 / 7,8 / 7), \\
0, & \text { otherwise. }\end{cases}
\end{gathered}
$$

It is easy to verify that $\phi_{1}$ and $\phi_{2}$ serve as scaling functions of a new GMRA $\left(U_{j}\right)$. However, since $\sigma_{\varphi_{1}}=\sigma_{\phi_{1}}=\chi_{\Omega_{1}}$ and $\sigma_{\varphi_{2}}=\sigma_{\phi_{2}}=\chi_{\Omega_{2}}$ where $\Omega_{1}=([-1 / 2,-3 / 7) \cup[-2 / 7,2 / 7) \cup[3 / 7,1 / 2))+\mathbb{Z}$ and $\Omega_{2}=[-1 / 7,1 / 7)+\mathbb{Z}$, we conclude that $\left(U_{j}\right)$ admits orthonormal wavelets.

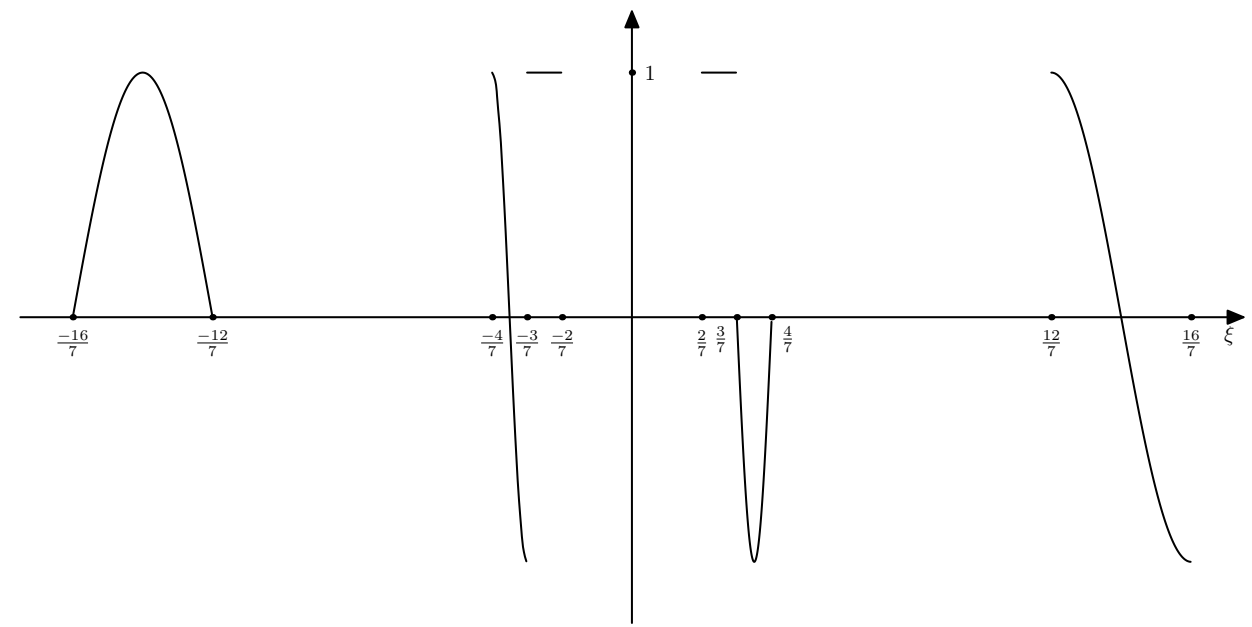

Fig. 2. The graph of $\hat{\psi}_{1}$

To obtain the resulting wavelet(s), we only need to find the filters and apply the technique of Remark 2.6. A short computation (the details are omitted) yields the Fourier transform of the associated wavelet $\psi_{1}$ :

$$
\hat{\psi}_{1}(\xi)= \begin{cases}a(\xi / 4), & \xi \in[-16 / 7,-12 / 7), \\ \overline{b(\xi)}, & \xi \in[-4 / 7,3 / 7), \\ 1, & \xi \in[-3 / 7,-2 / 7) \cup[2 / 7,3 / 7), \\ -\overline{a(\xi-1)}, & \xi \in[3 / 7,4 / 7), \\ b(\xi / 4-1), & \xi \in[12 / 7,16 / 7), \\ 0, & \text { otherwise. }\end{cases}
$$


Observe that $\psi_{1}$ is a non-MSF non-MRA wavelet with Journé's dimension function $D$. Note that some constructions (using different techniques) based on the Journé dimension function can be found in [4] and [5].

The function $\hat{\psi}_{1}$ with $a(\xi)=\sin ((7 \xi+4) \pi), b(\xi)=\cos ((7 \xi+4) \pi)$, $\xi \in(-4 / 7,-3 / 7)$ is shown in Figure 2.

3. From dimension functions to MSF wavelets. Let $D: \mathbb{R} \rightarrow$ $\mathbb{N} \cup\{0\}$ be an arbitrary dimension function. In this section we describe a method for construction of an MSF wavelet $\psi$ such that $D_{\psi}=D$ a.e. Throughout the section, the function $D$ will be fixed. We note that a similar construction (in $\mathbb{R}^{n}$ ) that uses a dimension function as the starting object appears in [11].

For the reader's convenience, we begin by restating Theorem 2 of [1]. That result characterizes dimension functions and will be repeatedly used in the course of our construction.

TheOREM 3.1. Let $D: \mathbb{R} \rightarrow \mathbb{N} \cup\{0\}$ be a measurable $\mathbb{Z}$-periodic function that is integrable on the unit interval $[-1 / 2,1 / 2)$. Then $D$ is the dimension function of some orthonormal wavelet if and only if the following conditions are satisfied:

(a) $\liminf _{j \rightarrow \infty} D\left(2^{-j} \xi\right) \geq 1$ a.e.;

(b) $D(\xi)+D(\xi+1 / 2)=D(2 \xi)+1$ a.e.;

(c) for a.e. $\xi$ such that $D(\xi) \geq 1$ there is $j \in \mathbb{N}$ satisfying $D\left(2^{-j} \xi+\right.$ $1 / 2) \geq 1$

(d) for a.e. $\xi$ such that $D(\xi) \geq 1$ there is $k \in \mathbb{Z}$ such that $D\left(2^{-j}(\xi+k)\right)$ $\geq 1$ for all $j \in \mathbb{Z}, j \geq 0$.

Basically, this theorem tells us that condition (3) of Theorem 4.2 in [12] is equivalent to conditions (c) and (d) above, provided that all other conditions are satisfied. Notice that $(\mathrm{d})$ means that for a.e. $\xi$ such that $D(\xi) \geq 1$ there exists at least one $k \in \mathbb{Z}$ such that $\xi+k \in \Delta$, where

$$
\Delta=\left\{\xi \in \mathbb{R}: D\left(2^{-j} \xi\right) \geq 1, \forall j \geq 0\right\} .
$$

Clearly, this is considerably weaker than (3) in Theorem 4.2 of [12] where one requires, for a.e. $\xi$ such that $D(\xi) \geq 1$, the existence of at least $D(\xi)$ integers $k$ with $\xi+k \in \Delta$.

Observe that all conditions of the above theorem are satisfied for almost all $\xi$ 's. By neglecting a null-set, we can suppose that these conditions are fulfilled for each point $\xi$ on the real line.

Define, for all $n \in \mathbb{N}$, the sets

$$
T_{n}=\{\xi \in[-1 / 2,1 / 2): D(\xi) \geq n\} .
$$

Denote $\Omega_{n}=T_{n}+\mathbb{Z}, n \in \mathbb{N}$. Observe that, since $D$ is a periodic function, 
$\Omega_{n}=\{\xi \in \mathbb{R}: D(\xi) \geq n\}$ and $T_{n}=\Omega_{n} \cap[-1 / 2,1 / 2)$. Obviously, we have $T_{1} \supseteq T_{2} \supseteq \cdots$ and $\Omega_{1} \supseteq \Omega_{2} \supseteq \cdots$. If $D$ is essentially bounded, say by $m$, we understand $T_{n}=\Omega_{n}=\emptyset$ for all $n>m$, and both sequences are considered to be finite.

REMARK 3.2. Our goal is to construct measurable functions $k_{n}: T_{n} \rightarrow \mathbb{Z}$, $n \in \mathbb{N}$, such that the sets defined by

$$
S_{n}:=\left\{\xi+k_{n}(\xi): \xi \in T_{n}\right\}, \quad n \in \mathbb{N},
$$

satisfy the conditions of Remark 2.2, so that the functions $\varphi_{n}$ defined by $\hat{\varphi_{n}}=\chi_{S_{n}}, n \in \mathbb{N}$, make up a scaling sequence for the $\operatorname{GMRA}\left(V_{j}\right)$, where $V_{0}=\bigoplus_{n=1}^{\infty}\left\langle\varphi_{n}\right\rangle$. Notice that the resulting $\operatorname{GMRA}\left(V_{j}\right)$ will be automatically admissible. Namely, $S_{n} \equiv T_{n}$ implies $S_{n}+\mathbb{Z}=T_{n}+\mathbb{Z}=\Omega_{n}=\{\xi \in \mathbb{R}$ : $D(\xi) \geq n\}$ for all $n \in \mathbb{N}$. From this we conclude that $\sum_{n=1}^{\infty} \chi_{S_{n}+\mathbb{Z}}(\xi)=$ $\sum_{n=1}^{\infty} \chi_{\Omega_{n}}(\xi)=\sum_{n=1}^{\infty} \chi_{\{\eta \in \mathbb{R}: D(\eta) \geq n\}}(\xi)=D(\xi)$. Since it is known that $D$ is finite a.e. and satisfies (b) of Theorem 3.1. we immediately conclude that both conditions of Remark 2.5 are satisfied as well.

We proceed by imposing certain conditions on the functions $\left(k_{n}\right)$ in order to ensure the conditions of Remark 2.2 (in other words, to ensure that the sequence $\left(\varphi_{n}\right)$ with $\hat{\varphi_{n}}=\chi_{S_{n}}$ is a scaling sequence for a GMRA).

Let us first rewrite the consistency equation (b) of Theorem 3.1 with $\xi$ replaced by $\xi / 2$ :

$$
D(\xi / 2)+D(\xi / 2+1 / 2)=D(\xi)+1 .
$$

Since $D$ is a periodic function, the second term on the left hand side may also be written as $D(\xi / 2-1 / 2)$. As our functions $k_{n}$ are going to be defined inside the unit interval, we shall need to know, for $\xi \in[-1 / 2,1 / 2)$, which of the points $\xi / 2-1 / 2, \xi / 2+1 / 2$ belongs to $[-1 / 2,1 / 2)$. Observe that the right choice is precisely $(\xi-\operatorname{sgn} \xi) / 2($ with the convention $\operatorname{sgn} 0=1$ ).

Let us now suppose that the functions $k_{n}: T_{n} \rightarrow \mathbb{Z}$ have already been constructed. For $\xi \in T_{1}$ and $n \in \mathbb{N}$ we introduce the following notations:

$$
\begin{aligned}
l_{n}(\xi) & =\min \{n, D(\xi / 2)\}, \quad r_{n}(\xi)=\min \{n, D((\xi-\operatorname{sgn} \xi) / 2)\}, \\
L_{n}(\xi) & =\left\{2 k_{i}(\xi / 2): i=1, \ldots, l_{n}(\xi)\right\}, \\
R_{n}(\xi) & =\left\{2 k_{j}((\xi-\operatorname{sgn} \xi) / 2)-\operatorname{sgn} \xi: j=1, \ldots, r_{n}(\xi)\right\}, \\
K_{n}(\xi) & =L_{n}(\xi) \cup R_{n}(\xi) .
\end{aligned}
$$

We understand that in the case $l_{n}(\xi)=0$ the set $L_{n}(\xi)$ is empty and, similarly, if $r_{n}(\xi)=0$ the set $R_{n}(\xi)$ is empty. However, by the consistency equation (3.3), for each $\xi$ in the unit interval, we have $D(\xi / 2) \geq 1$ or $D((\xi-\operatorname{sgn} \xi) / 2) \geq 1$. Hence, at least one of the numbers $l_{n}(\xi)$ and $r_{n}(\xi)$ must be positive, and consequently $K_{n}(\xi)$ is non-empty for every $\xi \in T_{1}$. 
Next we check that the numbers $2 k_{i}(\xi / 2), i=1, \ldots, l_{n}(\xi)$, resp. $2 k_{j}((\xi-\operatorname{sgn} \xi) / 2)-\operatorname{sgn} \xi, j=1, \ldots, r_{n}(\xi)$, make sense for every $\xi \in T_{1}$ such that $l_{n}(\xi) \geq 1$, resp. $r_{n}(\xi) \geq 1$. Suppose that $l_{n}(\xi) \geq 1$. Since $\xi / 2 \in$ $[-1 / 2,1 / 2)$ and $D(\xi / 2) \geq l_{n}(\xi)$, we have $\xi / 2 \in T_{i}$ for $i=1, \ldots, l_{n}(\xi)$, i.e., $\xi / 2$ belongs to the domains of $k_{i}, i=1, \ldots, l_{n}(\xi)$. Hence, the expressions $2 k_{i}(\xi / 2), i=1, \ldots, l_{n}(\xi)$, make sense. In a similar way one concludes that the integers $2 k_{j}((\xi-\operatorname{sgn} \xi) / 2)-\operatorname{sgn} \xi, j=1, \ldots, r_{n}(\xi)$, are well defined whenever $r_{n}(\xi) \geq 1$.

Finally, it is clear that $K_{n}(\xi) \subseteq K_{n+1}(\xi)$ for all $n \in \mathbb{N}$ and $\xi \in T_{1}$.

The sets $K_{n}(\xi)$ will play a central role in verifying that the sequence $\left(S_{n}\right)$ satisfies the conditions of Remark 2.2. Let us begin with two auxiliary results.

Lemma 3.3. Let $k_{n}: T_{n} \rightarrow \mathbb{Z}$ and $S_{n}=\left\{\xi+k_{n}(\xi): \xi \in T_{n}\right\}, n \in \mathbb{N}$. If $\xi \in[-1 / 2,1 / 2)$ and $k \in \mathbb{Z}$ are such that $\frac{1}{2}(\xi+k) \in S_{j}$ for some $j \in \mathbb{N}$, then $k=2 k_{j}(\xi / 2)$ or $k=2 k_{j}((\xi-\operatorname{sgn} \xi) / 2)-\operatorname{sgn} \xi$. In particular, $k \in K_{j}(\xi)$.

Proof. Since $\frac{1}{2}(\xi+k) \in S_{j}$, there is $\xi^{\prime} \in T_{j}$ such that $\frac{1}{2}(\xi+k)=\xi^{\prime}+k_{j}\left(\xi^{\prime}\right)$.

Suppose first that $k=2 l$ for some $l \in \mathbb{Z}$. Then $\xi / 2+l=\xi^{\prime}+k_{j}\left(\xi^{\prime}\right)$, hence $\xi / 2-\xi^{\prime}=k_{j}\left(\xi^{\prime}\right)-l \in \mathbb{Z}$. However, $\xi / 2-\xi^{\prime} \in(-3 / 4,3 / 4)$ yields $\xi / 2-\xi^{\prime}=0$, i.e., $\xi^{\prime}=\xi / 2$. We conclude that $l=k_{j}\left(\xi^{\prime}\right)=k_{j}(\xi / 2)$, so $k=2 k_{j}(\xi / 2)$. Since $\xi / 2 \in T_{j}$, we have $l_{j}(\xi)=\min \{j, D(\xi / 2)\}=j$, which implies $k \in K_{j}(\xi)$.

Suppose now that $k=2 l-\operatorname{sgn} \xi$ for some $l \in \mathbb{Z}$. Then $\xi / 2+l-(\operatorname{sgn} \xi) / 2=$ $\xi^{\prime}+k_{j}\left(\xi^{\prime}\right)$, so $(\xi-\operatorname{sgn} \xi) / 2-\xi^{\prime} \in \mathbb{Z}$. Furthermore, $(\xi-\operatorname{sgn} \xi) / 2-\xi^{\prime} \in(-1,1)$, yielding $(\xi-\operatorname{sgn} \xi) / 2-\xi^{\prime}=0$. Hence, $l=k_{j}\left(\xi^{\prime}\right)=k_{j}((\xi-\operatorname{sgn} \xi) / 2)$, so $k=2 k_{j}((\xi-\operatorname{sgn} \xi) / 2)-\operatorname{sgn} \xi$. Also, $(\xi-\operatorname{sgn} \xi) / 2 \in T_{j}$ implies $r_{j}(\xi)=$ $\min \{j, D((\xi-\operatorname{sgn} \xi) / 2)\}=j$; thus, $k \in K_{j}(\xi)$.

Lemma 3.4. Let $k_{n}: T_{n} \rightarrow \mathbb{Z}$ and $S_{n}=\left\{\xi+k_{n}(\xi): \xi \in T_{n}\right\}, n \in \mathbb{N}$.

(a) Let $i<j$. Then $S_{i} \cap S_{j}=\emptyset$ if and only if $k_{i}(\xi) \neq k_{j}(\xi)$ for all $\xi \in T_{j}$.

(b) $\frac{1}{2} S_{n} \subseteq S_{1} \cup \cdots \cup S_{n}$ if and only if $k_{n}(\xi) \in K_{n}(\xi)$ for all $\xi \in T_{n}$.

Proof. (a) Suppose that $k_{i}(\xi) \neq k_{j}(\xi)$ for all $\xi \in T_{j}$, and there is $\zeta \in$ $S_{i} \cap S_{j}$. Then there are $\xi \in T_{i}$ and $\eta \in T_{j}$ such that $\zeta=\xi+k_{i}(\xi)=$ $\eta+k_{j}(\eta)$. It follows that $\xi-\eta=k_{j}(\eta)-k_{i}(\xi)$. Since $\xi, \eta \in[-1 / 2,1 / 2)$ and $k_{i}(\xi), k_{j}(\eta) \in \mathbb{Z}$, we have $\xi-\eta=k_{j}(\eta)-k_{i}(\xi) \in(-1,1) \cap \mathbb{Z}=\{0\}$. Therefore, $\xi=\eta$ and $k_{i}(\xi)=k_{j}(\xi)$, which is not the case. Hence, $S_{i} \cap S_{j}=\emptyset$. The converse is obvious.

(b) First, suppose that $\frac{1}{2} S_{n} \subseteq S_{1} \cup \cdots \cup S_{n}$. Let $\xi \in T_{n}$. Then $\frac{1}{2}(\xi+$ $\left.k_{n}(\xi)\right) \in S_{j}$ for some $j \in\{1, \ldots, n\}$. By Lemma 3.3. $k_{n}(\xi) \in K_{j}(\xi) \subseteq K_{n}(\xi)$.

Conversely, suppose that $k_{n}(\xi) \in K_{n}(\xi)$ for all $\xi \in T_{n}$. Let $\zeta \in \frac{1}{2} S_{n}$. Then there is $\xi \in T_{n}$ such that $\zeta=\frac{1}{2}\left(\xi+k_{n}(\xi)\right)$. 
If $k_{n}(\xi)=2 k_{i}(\xi / 2)$ for some $i \in\left\{1, \ldots, l_{n}(\xi)\right\}$, then $\xi / 2 \in T_{i}$ and

$$
\zeta=\frac{1}{2}\left(\xi+2 k_{i}(\xi / 2)\right)=\xi / 2+k_{i}(\xi / 2) \in S_{i} \subseteq \bigcup_{j=1}^{n} S_{j} .
$$

Similarly, if $k_{n}(\xi)=2 k_{i}((\xi-\operatorname{sgn} \xi) / 2)-\operatorname{sgn} \xi$ for some $i \in\left\{1, \ldots, r_{n}(\xi)\right\}$, then $(\xi-\operatorname{sgn} \xi) / 2 \in T_{i}$ and

$$
\begin{aligned}
\zeta & =\frac{1}{2}\left(\xi+2 k_{i}\left(\frac{\xi-\operatorname{sgn} \xi}{2}\right)-\operatorname{sgn} \xi\right) \\
& =\frac{\xi-\operatorname{sgn} \xi}{2}+k_{i}\left(\frac{\xi-\operatorname{sgn} \xi}{2}\right) \in S_{i} \subseteq \bigcup_{j=1}^{n} S_{j} .
\end{aligned}
$$

Thus, $\frac{1}{2} S_{n} \subseteq S_{1} \cup \cdots \cup S_{n}$.

We are now ready to give sufficient conditions on functions $\left(k_{n}\right)$ in order to obtain a desired admissible GMRA.

Theorem 3.5. Let $D$ be a dimension function, $\Omega_{n}=\{\xi \in \mathbb{R}: D(\xi) \geq n\}$ and $T_{n}=\Omega_{n} \cap[-1 / 2,1 / 2), n \in \mathbb{N}$. Suppose that $\left(k_{n}\right)$ is a sequence of measurable functions $k_{n}: T_{n} \rightarrow \mathbb{Z}$ with the properties:

(a) $k_{i}(\xi) \neq k_{j}(\xi)$ for a.e. $\xi \in T_{j}$ and all $i<j$;

(b) $k_{n}(\xi) \in K_{n}(\xi)$ for a.e. $\xi \in T_{n}$ and all $n \in \mathbb{N}$;

(c) $k_{1}(\xi)=0$ for all $\xi \in T_{1} \cap \Delta$.

Let $S_{n}=\left\{\xi+k_{n}(\xi): \xi \in T_{n}\right\}, n \in \mathbb{N}$. Then the sequence of functions $\left(\varphi_{n}\right)$ defined by $\hat{\varphi_{n}}=\chi_{S_{n}}$ is a scaling sequence for an admissible GMRA. In particular, an associated wavelet $\psi$ is given by $\hat{\psi}=\chi_{2 S \backslash S}$, where $S=$ $\bigcup_{i=1}^{\infty} S_{i}$, and satisfies $D_{\psi}(\xi)=D(\xi)$ for a.e. $\xi \in \mathbb{R}$.

Proof. We need to check the conditions of Remark 2.2. Condition (1) follows from (a) and the first statement of the preceding lemma. Since, for each $n \in \mathbb{N}$, we have $S_{n} \equiv T_{n}$ and $\left|T_{n} \cap\left(T_{n}+k\right)\right|=0$ for all $k \in \mathbb{Z} \backslash\{0\}$, condition (2) is satisfied as well. Condition (3) follows from (b) and Lemma 3.4(b). Further, recall from [12] that each dimension function $D$ satisfies $\int_{-1 / 2}^{1 / 2} D(\xi) d \xi=1$. Since $\left|S_{n}\right|=\left|T_{n}\right|$ for all $n \in \mathbb{N}$, it follows that $\sum_{n=1}^{\infty}\left|S_{n}\right|=$ $\sum_{n=1}^{\infty}\left|T_{n}\right|=\int_{-1 / 2}^{1 / 2} D(\xi) d \xi=1$, which shows that (4) is fulfilled too.

It remains to verify (5). Pick $\xi \in \mathbb{R}$. First, let $j_{0}$ be the smallest nonnegative integer for which $\left(1 / 2^{j_{0}}\right) \xi \in[-1 / 2,1 / 2)$. By Theorem 3.1(a) applied to the point $\left(1 / 2^{j_{0}}\right) \xi$, there exists a non-negative integer $k_{0}$ such that $D\left(\frac{1}{2^{k}} \frac{1}{2^{j_{0}}} \xi\right) \geq 1$ for all $k \geq k_{0}$. In particular, $\frac{1}{2^{k_{0}}} \frac{1}{2^{j_{0}}} \xi \in T_{1} \cap \Delta$; thus, by (c), we have $\frac{1}{2^{k_{0}}} \frac{1}{2^{j_{0}}} \xi \in S_{1}$. This gives us condition (5) of Remark 2.2 with $m(\xi)=k_{0}+j_{0}$. 
We have already noted in Remark 3.2 that the resulting GMRA is admissible. From Proposition 2.7 we know that an associated wavelet $\psi$ is given by $\hat{\psi}=\chi_{2 S \backslash S}$. Finally, combining Remark 3.2 and the last assertion of Remark 2.5, we conclude $D_{\psi}=\sum_{n=1}^{\infty} \chi_{S_{n}+\mathbb{Z}}=D$.

REMARK 3.6. (a) Observe that under the assumptions of the preceding theorem we end up with a sequence $\left(S_{n}\right)$ with $\frac{1}{2} S_{n} \subseteq \bigcup_{i=1}^{n} S_{i}$, which is stronger than condition (3) in Remark 2.2. $\frac{1}{2} S_{n} \subseteq \bigcup_{i=1}^{\infty} S_{i}$.

(b) It is also useful to note that $\frac{1}{2} S_{n} \subseteq \bigcup_{i=1}^{\infty} S_{i}$ implies $S_{n} \subseteq \Delta$ for all $n \in \mathbb{N}$. Indeed, for every $\xi \in S_{n}$ and $i \in \mathbb{N} \cup\{0\}$ there is $k_{i} \in \mathbb{N}$ such that $2^{-i} \xi \in S_{k_{i}}$. Therefore, $D\left(2^{-i} \xi\right) \geq 1$ for all $i \in \mathbb{N} \cup\{0\}$.

(c) One can think of our construction of $\left(k_{n}\right)$ as finding the minimal integer translation $S_{n}$ of each $T_{n}$ such that the sets $S_{n}$ are pairwise disjoint and lie in $\Delta$. Observe that the assumption (c) in the preceding theorem that poses a condition on $k_{1}$ is in accordance with that principle. This would mean that our construction yields, for a given dimension function, a wavelet set with the smallest possible diameter. This is demonstrated in Example 3.19 for the Journé dimension function. However, we have not been able to prove this in full generality.

The rest of the section is devoted to a construction of a sequence $\left(k_{n}\right)$ that satisfies the conditions of Theorem 3.5. Let us begin by constructing $k_{1}$.

Lemma 3.7. Let $D: \mathbb{R} \rightarrow \mathbb{N} \cup\{0\}$ be a dimension function. For every $\xi \in T_{1} \backslash\{-1 / 2\}$ let $k_{1}(\xi) \in \mathbb{Z}$ be such that

$$
\left|\xi+k_{1}(\xi)\right|=\min \{|\xi+k|: k \in \mathbb{Z} \text { such that } \xi+k \in \Delta\} .
$$

Then $\xi \mapsto k_{1}(\xi) \in \mathbb{Z}$ is a well-defined function which satisfies $k_{1}(\xi) \in K_{1}(\xi)$ for all $\xi \in T_{1} \backslash\{-1 / 2\}$.

Proof. Since $D$ is a dimension function, for every $\xi \in T_{1}$, by Theorem $3.1(\mathrm{~d})$, there is $k \in \mathbb{Z}$ such that $\xi+k \in \Delta$. Therefore, the set $\{|\xi+k|$ : $k \in \mathbb{Z}$ such that $\xi+k \in \Delta\}$ is non-empty and, obviously, has a minimum.

Let us show that the relation (3.8) uniquely determines $k_{1}(\xi)$ for every $\xi \in T_{1} \backslash\{-1 / 2\}$. Notice that $0 \in T_{1} \Rightarrow 0 \in \Delta \Rightarrow k_{1}(0)=0$. Now suppose that $\xi \in T_{1} \backslash\{0,-1 / 2\}$ is such that there are $l, m \in \mathbb{Z}$ satisfying $|\xi+l|=$ $|\xi+m|=\min \{|\xi+k|: k \in \mathbb{Z}$ such that $\xi+k \in \Delta\}$. Then $\xi+m= \pm(\xi+l)$. Observe that $\xi+m=-(\xi+l)$ implies $2 \xi=-m-l \in \mathbb{Z} \cap((-1,1) \backslash\{0\})$, so this case cannot happen. Thus, $\xi+m=\xi+l$, i.e., $m=l$, so $k_{1}(\xi)$ is uniquely determined on $T_{1} \backslash\{-1 / 2\}$.

It remains to show the second statement. Let $\xi \in T_{1} \backslash\{-1 / 2\}$. First, $k_{1}(0)=2 k_{1}(0 / 2)=0 \in K_{1}(0)$, so let $\xi \neq 0$.

Suppose that $k_{1}(\xi)$ is even. Since $\xi+k_{1}(\xi) \in \Delta$ and $D$ is $\mathbb{Z}$-periodic, it follows that $D(\xi / 2)=D\left(2^{-1}\left(\xi+k_{1}(\xi)\right)\right) \geq 1$. Hence, $\xi / 2 \in T_{1} \backslash\{-1 / 2\}$, so $k_{1}(\xi / 2)$ is well defined by (3.8). Also, $l_{1}(\xi)=1$ implies $2 k_{1}(\xi / 2) \in K_{1}(\xi)$. It is 
obvious that $\xi / 2+k_{1}(\xi) / 2 \in \Delta$. Moreover, if $k \in \mathbb{Z}$ is such that $\xi / 2+k \in \Delta$, then $\xi+2 k \in \Delta$. Now, (3.8) implies $\left|\xi+k_{1}(\xi)\right| \leq|\xi+2 k|$ for all $k \in \mathbb{Z}$ with $\xi / 2+k \in \Delta$, i.e., $\left|\xi / 2+k_{1}(\xi) / 2\right| \leq|\xi / 2+k|$ for all such $k$. We conclude that $k_{1}(\xi / 2)=k_{1}(\xi) / 2$, i.e., $k_{1}(\xi)=2 k_{1}(\xi / 2)$.

Suppose now that $k_{1}(\xi)$ is odd. Since $\xi+k_{1}(\xi) \in \Delta$ and $D$ is $\mathbb{Z}$-periodic, it follows that $D((\xi-\operatorname{sgn} \xi) / 2)=D\left(2^{-1}\left(\xi+k_{1}(\xi)\right)\right) \geq 1$. Therefore, $(\xi-\operatorname{sgn} \xi) / 2 \in T_{1} \backslash\{-1 / 2\}$, so $k_{1}((\xi-\operatorname{sgn} \xi) / 2)$ is well defined. Also, $r_{1}(\xi)$ $=1$ implies $2 k_{1}((\xi-\operatorname{sgn} \xi) / 2)-\operatorname{sgn} \xi \in K_{1}(\xi)$. Obviously, $\left(k_{1}(\xi)+\operatorname{sgn} \xi\right) / 2$ $\in \mathbb{Z}$, and $(\xi-\operatorname{sgn} \xi) / 2+\left(k_{1}(\xi)+\operatorname{sgn} \xi\right) / 2 \in \Delta$. Furthermore, for all $k \in \mathbb{Z}$ such that $(\xi-\operatorname{sgn} \xi) / 2+k \in \Delta$, we have $\xi+2 k-\operatorname{sgn} \xi \in \Delta$. Therefore, $\left|\xi+k_{1}(\xi)\right| \leq|\xi+2 k-\operatorname{sgn} \xi|$ for all $k \in \mathbb{Z}$ such that $(\xi-\operatorname{sgn} \xi) / 2+k \in \Delta$, i.e., $\left|(\xi-\operatorname{sgn} \xi) / 2+\left(k_{1}(\xi)+\operatorname{sgn} \xi\right) / 2\right| \leq|(\xi-\operatorname{sgn} \xi) / 2+k|$ for all $k \in \mathbb{Z}$ such that $(\xi-\operatorname{sgn} \xi) / 2+k \in \Delta$. It follows that $k_{1}((\xi-\operatorname{sgn} \xi) / 2)=\left(k_{1}(\xi)+\operatorname{sgn} \xi\right) / 2$, i.e., $k_{1}(\xi)=2 k_{1}((\xi-\operatorname{sgn} \xi) / 2)-\operatorname{sgn} \xi$.

REMARK 3.8. Notice that if $-1 / 2 \in T_{1}$, then $k_{1}(-1 / 2)$ does not have to be uniquely determined by (3.8). Namely, from the first part of the proof of Lemma 3.7 it is obvious that if $k_{0} \in \mathbb{Z}$ satisfies (3.8) then so does $1-k_{0} \in \mathbb{Z}$, and $k_{0} \neq 1-k_{0}$. Without loss of generality we can assume that $k_{1}(-1 / 2)=k_{0}$ if $\left|k_{0}\right|<\left|1-k_{0}\right|$, and $k_{1}(-1 / 2)=1-k_{0}$ if $\left|k_{0}\right|>\left|1-k_{0}\right|$. Then the second part of the proof of Lemma 3.7 can be derived for $\xi=-1 / 2$, so $k_{1}(-1 / 2) \in K_{1}(-1 / 2)$.

It follows from Lemma 3.7 and the preceding remark that for every dimension function there is a function $k_{1}: T_{1} \rightarrow \mathbb{Z}$ satisfying (3.8) and such that $k_{1}(\xi) \in K_{1}(\xi)$ for every $\xi \in T_{1}$.

Theorem 3.9. Let $D: \mathbb{R} \rightarrow \mathbb{N} \cup\{0\}$ be a dimension function. Then there exist functions $k_{n}: T_{n} \rightarrow \mathbb{Z}, n \in \mathbb{N}$, satisfying the following conditions:

(a) $k_{i}(\xi) \neq k_{n}(\xi)$ for all $i<n$ and for every $\xi \in T_{n}, n \in \mathbb{N}$.

(b) $k_{n}(\xi) \in K_{n}(\xi)$ for each $n \in \mathbb{N}$ and every $\xi \in T_{n}$. Moreover, for each $n \geq 2$ and every $\xi \in T_{n}$ we have:

(b1) if $D(\xi / 2) \geq 1$ and $D((\xi-\operatorname{sgn} \xi) / 2) \geq 1$, then $k_{n}(\xi) \in K_{n-1}(\xi)$;

(b2) if $D(\xi / 2)=0$ or $D((\xi-\operatorname{sgn} \xi) / 2)=0$, then $k_{n}(\xi) \in K_{n}(\xi) \backslash$ $K_{n-1}(\xi)$. Furthermore, there are $m \in \mathbb{N}, \eta \in T_{n}$ and $p \in \mathbb{Z}$ such that $k_{n}(\xi)=2^{m} k_{n}(\eta)+p$ and $k_{n}(\eta) \in K_{n-1}(\eta)$.

(c) $k_{1}(\xi)=0$ for all $\xi \in T_{1} \cap \Delta$.

Proof. We prove the theorem by induction. Using Lemma 3.7 and Remark 3.8 we can construct $k_{1}$ which satisfies (b) and (c).

Let $n \geq 2$. Suppose that we have constructed functions $k_{i}: T_{i} \rightarrow \mathbb{Z}$, $i=1, \ldots, n-1$, satisfying (a) and (b). Let us construct $k_{n}: T_{n} \rightarrow \mathbb{Z}$. 
Let $\xi \in T_{n}$. Then $D(\xi / 2)+D((\xi-\operatorname{sgn} \xi) / 2)=D(\xi)+1 \geq n+1$. We have the following two cases:

(1) $D(\xi / 2) \geq 1$ and $D((\xi-\operatorname{sgn} \xi) / 2) \geq 1$,

(2) $D(\xi / 2)=0$ or $D((\xi-\operatorname{sgn} \xi) / 2)=0$.

(1) Suppose that $D(\xi / 2) \geq 1$ and $D((\xi-\operatorname{sgn} \xi) / 2) \geq 1$. We split the argument into three cases.

If $D(\xi / 2) \leq n-1$ and $D((\xi-\operatorname{sgn} \xi) / 2) \leq n-1$, then $l_{n-1}(\xi)=D(\xi / 2)$ and $r_{n-1}(\xi)=D((\xi-\operatorname{sgn} \xi) / 2)$.

If $D(\xi / 2) \geq n$, then $l_{n-1}(\xi)=n-1$ and $r_{n-1}(\xi) \geq 1$.

If $D((\xi-\operatorname{sgn} \xi) / 2) \geq n$, then $l_{n-1}(\xi) \geq 1$ and $r_{n-1}(\xi)=n-1$.

Notice that in each case $l_{n-1}(\xi)+r_{n-1}(\xi) \geq n$, so $K_{n-1}(\xi)$ contains at least $n$ different elements. By the inductive assumption we have $k_{1}(\xi)$, $\ldots, k_{n-1}(\xi) \in K_{n-1}(\xi)$. Therefore, $K_{n-1}(\xi) \backslash\left\{k_{1}(\xi), \ldots, k_{n-1}(\xi)\right\}$ is nonempty, so we can define $k_{n}(\xi)$ by

$$
\left|\xi+k_{n}(\xi)\right|=\min \left\{|\xi+k|: k \in K_{n-1}(\xi) \backslash\left\{k_{1}(\xi), \ldots, k_{n-1}(\xi)\right\}\right\} .
$$

(As in Lemma 3.7, it can be shown that this relation uniquely determines $k_{n}(\xi)$ if $\xi \in T_{n} \backslash\{-1 / 2\}$. Also, we define $k_{n}(-1 / 2)$ as in Remark 3.8.) It is obvious that (a) and (b) are satisfied.

(2) Now suppose that $D(\xi / 2)=0$ or $D((\xi-\operatorname{sgn} \xi) / 2)=0$.

First notice that for every $\xi^{\prime} \in T_{n}$ such that $D\left(\xi^{\prime} / 2\right)=0$ we have $K_{j}\left(\xi^{\prime}\right)=\left\{2 k_{i}\left(\left(\xi^{\prime}-\operatorname{sgn} \xi^{\prime}\right) / 2\right)-\operatorname{sgn} \xi^{\prime}: i=1, \ldots, j\right\}$ for all $j=1, \ldots, n-1$. Therefore, $k_{i}\left(\xi^{\prime}\right)=2 k_{i}\left(\left(\xi^{\prime}-\operatorname{sgn} \xi^{\prime}\right) / 2\right)-\operatorname{sgn} \xi^{\prime}$ for $i=1, \ldots, n-1$. Similarly, in the case $D\left(\left(\xi^{\prime}-\operatorname{sgn} \xi^{\prime}\right) / 2\right)=0$ we have $k_{i}\left(\xi^{\prime}\right)=2 k_{i}\left(\xi^{\prime} / 2\right)$ for $i=1, \ldots, n-1$. Hence,

$$
k_{i}\left(\xi^{\prime}\right)= \begin{cases}2 k_{i}\left(\xi^{\prime} / 2\right) & \text { if } D\left(\left(\xi^{\prime}-\operatorname{sgn} \xi^{\prime}\right) / 2\right)=0, \\ 2 k_{i}\left(\left(\xi^{\prime}-\operatorname{sgn} \xi^{\prime}\right) / 2\right)-\operatorname{sgn} \xi^{\prime} & \text { if } D\left(\xi^{\prime} / 2\right)=0,\end{cases}
$$

for all $i=1, \ldots, n-1$ and $\xi^{\prime} \in T_{n}$.

Since $D(\xi) \geq n \geq 1$, by Theorem 3.1(d) there is $q \in \mathbb{Z}$ such that $D\left(2^{-i}(\xi+q)\right) \geq 1$ for all $i \in \mathbb{N} \cup\{0\}$. By Theorem 3.1(c), there is $j \in \mathbb{N}$ such that $D\left(2^{-j}(\xi+q)+1 / 2\right) \geq 1$. Notice that $j \geq 2$, since $j=1$ implies $D(\xi / 2)$ $\geq 1$ and $D((\xi-\operatorname{sgn} \xi) / 2) \geq 1$, which is not the case. Suppose that we have chosen the smallest $j$ with the above property, i.e., $D\left(2^{-i}(\xi+q)+1 / 2\right)=0$ for $i=1, \ldots, j-1$.

Let $\xi_{i}=\tau\left(2^{-i}(\xi+q)\right)$ for all $i=0, \ldots, j-1$. Then $\xi_{0}=\xi$ and, for all $i=0, \ldots, j-2$, we have

$$
\xi_{i+1}=\frac{\xi_{i}}{2} \quad \text { or } \quad \xi_{i+1}=\frac{\xi_{i}-\operatorname{sgn} \xi_{i}}{2} .
$$


Since $D\left(\xi_{i}\right)=D\left(2^{-i}(\xi+q)\right) \geq 1$ for $i=0, \ldots, j-1$, it is clear that $D\left(\left(\xi_{i}-\operatorname{sgn} \xi_{i}\right) / 2\right)=0$ implies $\xi_{i+1}=\xi_{i} / 2$, while $D\left(\xi_{i} / 2\right)=0$ implies $\xi_{i+1}=\left(\xi_{i}-\operatorname{sgn} \xi_{i}\right) / 2$ for $i=0, \ldots, j-2$.

Conversely, if $\xi_{i+1}=\xi_{i} / 2$ then

$$
D\left(\frac{\xi_{i}-\operatorname{sgn} \xi_{i}}{2}\right)=D\left(\xi_{i+1}-\frac{\operatorname{sgn} \xi_{i}}{2}\right)=D\left(2^{-(i+1)}(\xi+q)+1 / 2\right)=0,
$$

while in the case $\xi_{i+1}=\left(\xi_{i}-\operatorname{sgn} \xi_{i}\right) / 2$,

$$
D\left(\frac{\xi_{i}}{2}\right)=D\left(\xi_{i+1}+\frac{\operatorname{sgn} \xi_{i}}{2}\right)=D\left(2^{-(i+1)}(\xi+q)+\frac{1}{2}\right)=0
$$

for $i=0, \ldots, j-2$. Therefore,

$$
\begin{aligned}
\xi_{i+1} & =\frac{\xi_{i}}{2} \Leftrightarrow D\left(\frac{\xi_{i}-\operatorname{sgn} \xi_{i}}{2}\right)=0, \\
\xi_{i+1} & =\frac{\xi_{i}-\operatorname{sgn} \xi_{i}}{2} \Leftrightarrow D\left(\frac{\xi_{i}}{2}\right)=0,
\end{aligned}
$$

for $i=0, \ldots, j-2$.

Moreover, for all $i=1, \ldots, j-1$ we have

$$
\begin{aligned}
D\left(\xi_{i}\right) & =D\left(\xi_{i}\right)+D\left(2^{-i}(\xi+q)+\frac{1}{2}\right) \\
& =D\left(\xi_{i}\right)+D\left(\xi_{i}+\frac{1}{2}\right)=D\left(2 \xi_{i}\right)+1=D\left(\xi_{i-1}\right)+1,
\end{aligned}
$$

so that $D\left(\xi_{i}\right) \geq n$, i.e., $\xi_{i} \in T_{n}$.

Notice that $\xi_{j-1}$ satisfies

$$
\begin{aligned}
& D\left(\xi_{j-1}\right) \geq n, \quad D\left(\frac{\xi_{j-1}}{2}\right)=D\left(2^{-j}(\xi+q)\right) \geq 1, \\
& D\left(\frac{\xi_{j-1}-\operatorname{sgn} \xi_{j-1}}{2}\right)=D\left(2^{-j}(\xi+q)+\frac{1}{2}\right) \geq 1,
\end{aligned}
$$

which is case (1). Therefore, $k_{n}\left(\xi_{j-1}\right) \in K_{n-1}\left(\xi_{j-1}\right)$ is defined by using (3.9). Now we define $k_{n}\left(\xi_{j-2}\right), k_{n}\left(\xi_{j-3}\right), \ldots, k_{n}\left(\xi_{1}\right)$ and finally $k_{n}\left(\xi_{0}\right)=k_{n}(\xi)$ by

$$
k_{n}\left(\xi_{j-i}\right)= \begin{cases}2 k_{n}\left(\xi_{j-i+1}\right) & \text { if } \xi_{j-i+1}=\xi_{j-i} / 2, \\ 2 k_{n}\left(\xi_{j-i+1}\right)-\operatorname{sgn} \xi_{j-i} & \text { if } \xi_{j-i+1}=\left(\xi_{j-i}-\operatorname{sgn} \xi_{j-i}\right) / 2,\end{cases}
$$

for all $i=2, \ldots, j$. In other words,

$$
\begin{aligned}
& k_{n}\left(\xi_{j-i}\right) \\
= & \begin{cases}2 k_{n}\left(\xi_{j-i} / 2\right) & \text { if } D\left(\left(\xi_{j-i}-\operatorname{sgn} \xi_{j-i}\right) / 2\right)=0, \\
2 k_{n}\left(\left(\xi_{j-i}-\operatorname{sgn} \xi_{j-i}\right) / 2\right)-\operatorname{sgn} \xi_{j-i} & \text { if } D\left(\xi_{j-i} / 2\right)=0,\end{cases}
\end{aligned}
$$

for all $i=2, \ldots, j$. 
Next we show that $k_{n}(\xi) \neq k_{i}(\xi)$ for $i=1, \ldots, n-1$. First, since $k_{n}\left(\xi_{j-1}\right)$ is defined by (3.9), we have $k_{n}\left(\xi_{j-1}\right) \neq k_{i}\left(\xi_{j-1}\right)$ for $i=1, \ldots, n-1$.

Suppose that $D\left(\xi_{j-2} / 2\right)=0$. By (3.11), $\xi_{j-1}=\left(\xi_{j-2}-\operatorname{sgn} \xi_{j-2}\right) / 2$, and by $(3.13), k_{n}\left(\xi_{j-2}\right)=2 k_{n}\left(\left(\xi_{j-2}-\operatorname{sgn} \xi_{j-2}\right) / 2\right)-\operatorname{sgn} \xi_{j-2}=2 k_{n}\left(\xi_{j-1}\right)-$ $\operatorname{sgn} \xi_{j-2}$. Also, by (3.10), $k_{i}\left(\xi_{j-2}\right)=2 k_{i}\left(\left(\xi_{j-2}-\operatorname{sgn} \xi_{j-2}\right) / 2\right)-\operatorname{sgn} \xi_{j-2}=$ $2 k_{i}\left(\xi_{j-1}\right)-\operatorname{sgn} \xi_{j-2}$ for $i=1, \ldots, n-1$. Finally, for all $i=1, \ldots, n-1$ we get

$$
\begin{aligned}
k_{n}\left(\xi_{j-1}\right) \neq k_{i}\left(\xi_{j-1}\right) & \Rightarrow 2 k_{n}\left(\xi_{j-1}\right)-\operatorname{sgn} \xi_{j-2} \neq 2 k_{i}\left(\xi_{j-1}\right)-\operatorname{sgn} \xi_{j-2} \\
& \Rightarrow k_{n}\left(\xi_{j-2}\right) \neq k_{i}\left(\xi_{j-2}\right) .
\end{aligned}
$$

The same conclusion is obtained in the case $D\left(\left(\xi_{j-2}-\operatorname{sgn} \xi_{j-2}\right) / 2\right)=0$. Similarly, $k_{n}\left(\xi_{j-3}\right) \neq k_{i}\left(\xi_{j-3}\right)$ for $i=1, \ldots, n-1$, etc. Finally, $k_{n}\left(\xi_{0}\right) \neq$ $k_{i}\left(\xi_{0}\right)$, i.e., $k_{n}(\xi) \neq k_{i}(\xi)$ for $i=1, \ldots, n-1$.

From this and (3.13) it now follows that $k_{n}(\xi) \in K_{n}(\xi) \backslash K_{n-1}(\xi)$.

It is easy to see that $k_{n}(\xi)=2^{j-1} k_{n}\left(\xi_{j-1}\right)+p$ for some $p \in \mathbb{Z}$. If we put $m:=j-1 \in \mathbb{N}, \eta:=\xi_{j-1}$, we get (b2).

REMARK 3.10. From the proof of Theorem 3.9 (i.e., 3.10) and (3.13) one can see that, for each $\xi \in T_{n}$ such that $D(\xi / 2)=0$ or $D((\xi-\operatorname{sgn} \xi) / 2)$ $=0$, we have

$$
k_{i}(\xi)= \begin{cases}2 k_{i}(\xi / 2) & \text { if } D((\xi-\operatorname{sgn} \xi) / 2)=0, \\ 2 k_{i}((\xi-\operatorname{sgn} \xi) / 2)-\operatorname{sgn} \xi & \text { if } D(\xi / 2)=0,\end{cases}
$$

for all $i=1, \ldots, n$.

REMARK 3.11. Observe that the sequence $\left(k_{n}\right)$ constructed in Lemma 3.7 and Theorem 3.9 satisfies conditions (a)-(c) of Theorem 3.5. Thus, in order to finish our construction we only need to show that each $k_{n}$ is a measurable function, i.e., $S_{n}=\left\{\xi+k_{n}(\xi): \xi \in T_{n}\right\}, n \in \mathbb{N}$, is a measurable set.

Recall that, by Lemma 3.4. $S_{i} \cap S_{j}=\emptyset$ for $i \neq j$, and $\frac{1}{2} S_{n} \subseteq \bigcup_{i=1}^{n} S_{i}$.

First we note the following simple consequence of Theorem 3.9 .

Corollary 3.12. Let $D: \mathbb{R} \rightarrow \mathbb{N} \cup\{0\}$ be a dimension function. Let $\xi \in S_{n+1}$. Then

(a) $\xi / 2 \in S_{1} \cup \cdots \cup S_{n}$ if and only if $D(\xi / 2+1 / 2) \geq 1$;

(b) $\xi / 2 \in S_{n+1}$ if and only if $D(\xi / 2+1 / 2)=0$;

(c) there is $j \in \mathbb{N}$ such that $\xi \in S_{n+1}, \ldots, \xi / 2^{j-1} \in S_{n+1}, \xi / 2^{j} \in S_{1} \cup$ $\cdots \cup S_{n}$.

Proof. Set $\xi=\xi^{\prime}+k_{n+1}\left(\xi^{\prime}\right)$ where $\xi^{\prime} \in T_{n+1}$.

(a) Suppose that $\xi / 2 \in S_{1} \cup \cdots \cup S_{n}$. If $D(\xi / 2+1 / 2)=0$, then $D\left(\xi^{\prime} / 2+\right.$ $1 / 2)=0$ or $D\left(\xi^{\prime} / 2\right)=0$. By Remark 3.10, it follows $k_{n+1}\left(\xi^{\prime}\right)=2 k_{n+1}\left(\xi^{\prime} / 2\right)$ 
or $k_{n+1}\left(\xi^{\prime}\right)=2 k_{n+1}\left(\left(\xi^{\prime}-\operatorname{sgn} \xi^{\prime}\right) / 2\right)-\operatorname{sgn} \xi^{\prime}$. But this contradicts the assumption, since in the first case we would get

$$
\frac{\xi}{2}=\frac{\xi^{\prime}+k_{n+1}\left(\xi^{\prime}\right)}{2}=\frac{\xi^{\prime}}{2}+k_{n+1}\left(\frac{\xi^{\prime}}{2}\right) \in S_{n+1},
$$

while in the second case

$$
\frac{\xi}{2}=\frac{\xi^{\prime}+k_{n+1}\left(\xi^{\prime}\right)}{2}=\frac{\xi^{\prime}-\operatorname{sgn} \xi^{\prime}}{2}+k_{n+1}\left(\frac{\xi^{\prime}-\operatorname{sgn} \xi^{\prime}}{2}\right) \in S_{n+1},
$$

which is impossible by Lemma 3.4 (a). Therefore, $D(\xi / 2+1 / 2) \geq 1$.

Conversely, suppose that $D(\xi / 2+1 / 2) \geq 1$. Since $\xi \in S_{n+1} \subseteq \Delta$ by Remark 3.6(b), we have $D(\xi / 2) \geq 1$. Hence, $D\left(\xi^{\prime} / 2\right) \geq 1$ and $D\left(\xi^{\prime} / 2+1 / 2\right)$ $\geq 1$, so by Theorem 3.9 (b1) there is $j \leq n$ such that $k_{n+1}\left(\xi^{\prime}\right)=2 k_{j}\left(\xi^{\prime} / 2\right)$ or $k_{n+1}\left(\xi^{\prime}\right)=2 k_{j}\left(\left(\xi^{\prime}-\operatorname{sgn} \xi^{\prime}\right) / 2\right)-\operatorname{sgn} \xi^{\prime}$. It follows that

$$
\frac{\xi}{2}=\frac{\xi^{\prime}+k_{n+1}\left(\xi^{\prime}\right)}{2}=\frac{\xi^{\prime}}{2}+k_{j}\left(\frac{\xi^{\prime}}{2}\right) \in S_{j}
$$

or

$$
\frac{\xi}{2}=\frac{\xi^{\prime}+k_{n+1}\left(\xi^{\prime}\right)}{2}=\frac{\xi^{\prime}-\operatorname{sgn} \xi^{\prime}}{2}+k_{j}\left(\frac{\xi^{\prime}-\operatorname{sgn} \xi^{\prime}}{2}\right) \in S_{j},
$$

which proves the statement.

(b) This follows directly from (a) and Lemma 3.4 .

(c) First, Lemma 3.4 (b) implies $\xi / 2^{j} \in S_{1} \cup \cdots \cup S_{n+1}$ for all $j \in \mathbb{N}$. If $\xi / 2^{j} \in S_{n+1}$ for all $j \in \mathbb{N}$, then (b) would imply that $D\left(2^{-j} \xi+1 / 2\right)=0$ for all $j \in \mathbb{N}$, which is impossible by Theorem 3.1(c). Therefore, there is $j \in \mathbb{N}$ such that $\xi / 2^{j} \notin S_{n+1}$. The smallest such $j$ is as desired.

We now turn to the proof of measurability of the functions $k_{n}$ constructed in Lemma 3.7 and Theorem 3.9.

LEMma 3.13. The function $k_{1}$ is measurable.

Proof. We must show that the sets $k_{1}^{-1}(\{n\})=\left\{\xi \in T_{1}: k_{1}(\xi)=n\right\}$ are measurable for all $n \in \mathbb{Z}$. First $k_{1}^{-1}(\{0\})=T_{1} \cap \Delta$ is measurable.

In general, for $\xi \in T_{1}$ such that $\xi>0$, the number $k_{1}(\xi)$ is the first $n$ in the sequence $0,-1,1,-2,2,-3,3, \ldots$ for which $\xi+n \in \Delta$. Analogously, if $\xi<0$, then $k_{1}(\xi)$ is the first $n$ in the sequence $0,1,-1,2,-2,3,-3, \ldots$ for which $\xi+n \in \Delta$. Therefore, for every $n \in \mathbb{Z} \backslash\{0\}$ we have: $k_{1}(\xi)=n$ if and only if the following conditions are satisfied:

(1) $\xi \in T_{1}$ and $\xi+n \in \Delta$,

(2) $\xi+k \notin \Delta$ for all $k=-|n-\operatorname{sgn} n|, \ldots,|n-\operatorname{sgn} n|$,

(3) if $\operatorname{sgn} \xi=\operatorname{sgn} n$ then $\xi-n \notin \Delta$. 
In other words,

$$
k_{1}^{-1}(\{n\})=\left(T_{1} \cap(\Delta-n)\right) \backslash \bigcup_{k=-|n-\operatorname{sgn} n|}^{|n-\operatorname{sgn} n|}(\Delta+k) \cup\{\xi \in \Delta+n: \operatorname{sgn} \xi=\operatorname{sgn} n\},
$$

so $k_{1}^{-1}(\{n\})$ is measurable.

To show that the sets $S_{n}, n \geq 2$, are measurable, for a fixed $n \in \mathbb{N}$ we introduce the following sets:

$$
B_{1}=\left\{\xi \in S_{n+1}: \xi / 2 \in S_{1} \cup \cdots \cup S_{n}\right\},
$$

and for $j \geq 2$,

$$
B_{j}=\left\{\xi \in S_{n+1}: 2^{-1} \xi \in S_{n+1}, \ldots, 2^{-(j-1)} \xi \in S_{n+1}, 2^{-j} \xi \in S_{1} \cup \cdots \cup S_{n}\right\} .
$$

By statements (a) and (b) of Corollary 3.12 , it is obvious that

$$
\begin{gathered}
B_{1}=\left\{\xi \in S_{n+1}: D(\xi / 2+1 / 2) \geq 1\right\}, \\
B_{j}=\left\{\xi \in S_{n+1}: D\left(2^{-1} \xi+1 / 2\right)=\cdots=D\left(2^{-(j-1)} \xi+1 / 2\right)=0,\right. \\
\left.D\left(2^{-j} \xi+1 / 2\right) \geq 1\right\}, \quad j \geq 2 .
\end{gathered}
$$

Moreover, by Corollary 3.12 (c), we have $S_{n+1}=\bigcup_{j \in \mathbb{N}} B_{j}$. Therefore it is enough to prove that the sets $B_{j}, j \in \mathbb{N}$, are measurable. The following result shows that it suffices to handle $B_{1}$.

Lemma 3.14. For each $j \geq 2$,

$$
B_{j}=\left\{\xi \in 2 B_{j-1}: D(\xi) \geq n+1, D(\xi / 2+1 / 2)=0\right\} .
$$

Proof. It follows from the definition that $\frac{1}{2} B_{j} \subseteq B_{j-1}$, so obviously

$$
B_{j} \subseteq\left\{\xi \in 2 B_{j-1}: D(\xi) \geq n+1, D(\xi / 2+1 / 2)=0\right\} .
$$

Conversely, take $\xi \in 2 B_{j-1}$ such that $D(\xi) \geq n+1$ and $D(\xi / 2+1 / 2)=0$. Then $\xi / 2 \in B_{j-1}$, so $D\left(2^{-1} \xi+1 / 2\right)=\cdots=D\left(2^{-(j-1)} \xi+1 / 2\right)=0$ and $D\left(2^{-j} \xi+1 / 2\right) \geq 1$. To prove that $\xi \in B_{j}$, it is enough to check that $\xi \in S_{n+1}$. Since $D(\xi) \geq n+1$, we can write $\xi=\xi^{\prime}+k$, where $\xi^{\prime} \in T_{n+1}$ and $k \in \mathbb{Z}$. Then $\left(\xi^{\prime}+k\right) / 2=\xi / 2 \in S_{n+1}$; hence, by Lemma $3.3, k=2 k_{n+1}\left(\xi^{\prime} / 2\right)$ or $k=2 k_{n+1}\left(\left(\xi^{\prime}-\operatorname{sgn} \xi^{\prime}\right) / 2\right)-\operatorname{sgn} \xi^{\prime}$.

In the first case $\left(\xi-\xi^{\prime}\right) / 2=k / 2=k_{n+1}\left(\xi^{\prime} / 2\right) \in \mathbb{Z}$, so the $\mathbb{Z}$-periodicity of $D$ yields $D\left(\xi^{\prime} / 2+1 / 2\right)=D(\xi / 2+1 / 2)=0$. By Remark 3.10, we have $k_{n+1}\left(\xi^{\prime}\right)=2 k_{n+1}\left(\xi^{\prime} / 2\right)=k$. So, $\xi=\xi^{\prime}+k=\xi^{\prime}+k_{n+1}\left(\xi^{\prime}\right) \in S_{n+1}$.

Suppose now that $k=2 k_{n+1}\left(\left(\xi^{\prime}-\operatorname{sgn} \xi^{\prime}\right) / 2\right)-\operatorname{sgn} \xi^{\prime}$. Then

$$
\left(\left(\xi+\operatorname{sgn} \xi^{\prime}\right)-\xi^{\prime}\right) / 2=k / 2+\left(\operatorname{sgn} \xi^{\prime}\right) / 2=k_{n+1}\left(\left(\xi^{\prime}-\operatorname{sgn} \xi^{\prime}\right) / 2\right) \in \mathbb{Z},
$$

so $D\left(\xi^{\prime} / 2\right)=D\left(\left(\xi+\operatorname{sgn} \xi^{\prime}\right) / 2\right)=D(\xi / 2+1 / 2)=0$. Using Remark 3.10 . we see that $k_{n+1}\left(\xi^{\prime}\right)=2 k_{n+1}\left(\left(\xi^{\prime}-\operatorname{sgn} \xi^{\prime}\right) / 2\right)-\operatorname{sgn} \xi^{\prime}=k$, i.e., $\xi=\xi^{\prime}+k=$ $\xi^{\prime}+k_{n+1}\left(\xi^{\prime}\right) \in S_{n+1}$. This proves our statement.

Proposition 3.15. The sets $S_{n}, n \in \mathbb{N}$, are measurable. 
Proof. We prove the statement by induction. The set $S_{1}$ is measurable by Lemma 3.13 . Suppose that $S_{1}, \ldots, S_{n}$ are measurable for some $n \in \mathbb{N}$. To show that $S_{n+1}$ is also measurable, set

$$
\begin{aligned}
& A=2\left(S_{1} \cup \cdots \cup S_{n}\right) \backslash\left(S_{1} \cup \cdots \cup S_{n}\right), \\
& C=\left\{\xi \in T_{n+1}: D(\xi / 2) \geq 1, D(\xi / 2+1 / 2) \geq 1\right\} .
\end{aligned}
$$

For each $\xi \in C$, let $k(\xi) \in \mathbb{Z}$ be such that

$$
|\xi+k(\xi)|=\min \{|\xi+k|: k \in \mathbb{Z} \text { such that } \xi+k \in A\} .
$$

As in the proof of Lemma 3.7 (see also Remark 3.8), $\xi \mapsto k(\xi)$ is a well-defined function on $C$. Since $A$ and $C$ are measurable, so is

$$
G=\{\xi+k(\xi): \xi \in C\},
$$

being defined in the same way as $S_{1}$, already proved to be measurable. (Notice that the roles of the sets $T_{1}$ and $\Delta$ from the definition of $S_{1}$ are now taken by the sets $C$ and $A$, respectively.) Let us show that $B_{1}=G$.

First, let $\eta \in B_{1}$. Then $\eta=\xi+k_{n+1}(\xi)$, where $\xi \in T_{n+1}$. Also, $D(\eta / 2) \geq 1$ and $D(\eta / 2+1 / 2) \geq 1$, which implies $D(\xi / 2) \geq 1$ and $D(\xi / 2+1 / 2) \geq 1$. Hence, $\xi \in C$. To prove that $\eta \in G$, we must show that $k_{n+1}(\xi)=k(\xi)$. So, let $k \in \mathbb{Z}$ be such that $\xi+k \in A$. Then $\frac{1}{2}(\xi+k) \in S_{1} \cup \cdots \cup S_{n}$, so $k \in K_{n}(\xi)$ by Lemma 3.3. However, $\xi+k \notin S_{1} \cup \cdots \cup S_{n}$, so $k \in K_{n}(\xi) \backslash$ $\left\{k_{1}(\xi), \ldots, k_{n}(\xi)\right\}$. Therefore, the definition of $k_{n+1}: T_{n+1} \rightarrow \mathbb{Z}$ (formula (3.9) ) yields $\left|\xi+k_{n+1}(\xi)\right| \leq|\xi+k|$. Also, $\xi+k_{n+1}(\xi)=\eta \in B_{1} \subseteq A$. We conclude that $k_{n+1}(\xi)=k(\xi)$; hence, $\eta \in G$. Thus, $B_{1} \subseteq G$.

Conversely, let $\xi \in C$. Since $D(\xi / 2) \geq 1$ and $D(\xi / 2+1 / 2) \geq 1$, we get $D\left(\left(\xi+k_{n+1}(\xi)\right) / 2+1 / 2\right) \geq 1$, so $\xi+k_{n+1}(\xi) \in B_{1} \subseteq G$. Thus, $k_{n+1}(\xi)=$ $k(\xi)$, which implies $\xi+k(\xi)=\xi+k_{n+1}(\xi) \in B_{1}$. Therefore, $G \subseteq B_{1}$.

In particular, $B_{1}$ is measurable. Since $D$ is a measurable function, it follows inductively from Lemma 3.14 that all $B_{j}, j \in \mathbb{N}$, are measurable. By Corollary 3.12 (c), we have $S_{n+1}=\bigcup_{j \in \mathbb{N}} B_{j}$; hence, $S_{n+1}$ is also measurable.

This completes our argument. Notice that our entire construction of an MSF wavelet from a given dimension function is described in Theorem 3.5 , Lemma 3.7, and (the proof of) Theorem 3.9

A natural question is whether it is possible to find simpler formulae for $\left(k_{n}\right)$. The answer is negative since a thorough examination of the proof of Theorem 3.9 shows that the process of defining the value of $k_{n}$ at a point $\xi$ depends on $D(\xi / 2)$ and $D(\xi / 2+1 / 2)$. Thus, a certain discussion depending on those two numbers is unavoidable.

However, the construction becomes easier and more transparent if we impose some additional conditions on the starting dimension function $D$. In 
our last proposition we shall review the preceding construction under the additional assumption that $D(\xi) \geq 1$ for all $\xi \in[-1 / 4,0)$.

Lemma 3.16. Let $D: \mathbb{R} \rightarrow \mathbb{N} \cup\{0\}$ be a dimension function that is positive on $[-1 / 4,0)$. For $\xi \in T_{1}$, let $j(\xi)$ be the smallest natural number with $D\left(2^{-j(\xi)} \xi-1 / 2\right) \geq 1$. Then the function $k_{1}: T_{1} \rightarrow \mathbb{Z}$ defined by (3.8) is given by

$$
k_{1}(\xi)= \begin{cases}0, & \xi \in T_{1} \cap \Delta, \\ -2^{j(\xi)-1}, & \xi \in T_{1} \backslash \Delta .\end{cases}
$$

Proof. First, $j(\xi)$ is well defined by Proposition 1 of [1].

Observe that $\xi \in \Delta$ for all $\xi \in T_{1} \cap[-1 / 2,0)$. For such $\xi$ we have $k_{1}(\xi)=0$.

Now take $\xi \in T_{1} \cap[0,1 / 2)$. If $\xi \in \Delta$, then $k_{1}(\xi)=0$, so the assertion is true.

Let $\xi \notin \Delta$. Observe that $\xi-2^{j(\xi)-1} \in \Delta$. Indeed, if $j(\xi) \geq 2$, then, by the choice of $j(\xi)$ we have $D\left(2^{-j} \xi-1 / 2\right)=0$ for all $j=1, \ldots, j(\xi)-1$; thus,

$$
D\left(2^{-j} \xi\right)=D\left(2^{-j} \xi\right)+D\left(2^{-j} \xi-1 / 2\right)=D\left(2^{-(j-1)} \xi\right)+1 \geq 1 .
$$

Then we get

$$
D\left(2^{-j}\left(\xi-2^{j(\xi)-1}\right)\right)=D\left(2^{-j} \xi\right) \geq 1
$$

for $j=0, \ldots, j(\xi)-1$. Furthermore,

$$
D\left(2^{-j(\xi)}\left(\xi-2^{j(\xi)-1}\right)\right)=D\left(2^{-j(\xi)} \xi-1 / 2\right) \geq 1,
$$

while for $j>j(\xi)$ we have $2^{-j}\left(\xi-2^{j(\xi)-1}\right) \in[-1 / 4,0)$, so $D\left(2^{-j}(\xi-\right.$ $\left.\left.2^{j(\xi)-1}\right)\right) \geq 1$ by assumption. Thus, $\xi-2^{j(\xi)-1} \in \Delta$.

It remains to prove that $\left|\xi-2^{j(\xi)-1}\right| \leq|\xi+k|$ for all $k \in \mathbb{Z}$ such that $\xi+k \in \Delta$. Fix such a $k$. (Notice that $k \neq 0$, since $\xi \notin \Delta$.) First, we show that $|k| \geq 2^{j(\xi)-1}$. This is obvious if $j(\xi)=1$, so let $j(\xi) \geq 2$. If $2^{-j} k+1 / 2=2^{-j}(\xi+k)-\left(2^{-j} \xi-1 / 2\right) \in \mathbb{Z}$ for some $j=1, \ldots, j(\xi)-1$, we would have $D\left(2^{-j}(\xi+k)\right)=D\left(2^{-j} \xi-1 / 2\right)=0$, which is impossible since $\xi+k \in \Delta$. Therefore, $2^{-j} k+1 / 2 \notin \mathbb{Z}$ for all $j=1, \ldots, j(\xi)-1$, so $k \neq 2^{j-1}(2 l+1)$ for all $j=1, \ldots, j(\xi)-1$ and $l \in \mathbb{Z}$. We conclude that $|k| \neq 2^{j}(2 l+1)$ for all $j=0, \ldots, j(\xi)-2$ and $l \in \mathbb{N} \cup\{0\}$. Hence, $|k| \geq 2^{j(\xi)-1}$. Now we have

$$
\left|\xi-2^{j(\xi)-1}\right|=-\xi+2^{j(\xi)-1} \leq-\xi+|k|=|k|-|\xi| \leq|\xi+k| ;
$$

hence, $k_{1}(\xi)=-2^{j(\xi)-1}$ and the proof is complete.

Proposition 3.17. Let $D: \mathbb{R} \rightarrow \mathbb{N} \cup\{0\}$ be a dimension function that is positive on $[-1 / 4,0)$. For $\xi \in T_{1}$ let $j(\xi)$ be the smallest natural number with $D\left(2^{-j(\xi)} \xi-1 / 2\right) \geq 1$. If $D\left(2^{-j(\xi)} \xi\right)=0$, let $n(\xi)$ be the smallest natural 
number with $D\left(2^{-n(\xi)}\left(2^{-j(\xi)} \xi-1 / 2\right)+1 / 2\right) \geq 1$. Then the functions $k_{n}$ : $T_{n} \rightarrow \mathbb{Z}, n \geq 2$, constructed in Theorem 3.9 are given by

$k_{n}(\xi)= \begin{cases}2^{j(\xi)-1} k_{n}\left(2^{-j(\xi)+1} \xi\right) & \text { if } D\left(2^{-j(\xi)} \xi\right) \geq 1, \\ 2^{j(\xi)+n(\xi)-1} k_{n}\left(2^{-j(\xi)-n(\xi)+1} \xi-2^{-n(\xi)}\right)-2^{j(\xi)-1} & \text { if } D\left(2^{-j(\xi)} \xi\right)=0 .\end{cases}$

Moreover, if $D\left(2^{-j(\xi)} \xi\right) \geq 1$, then

$$
k_{n}\left(2^{-j(\xi)+1} \xi\right) \in K_{n-1}\left(2^{-j(\xi)+1} \xi\right) \backslash\left\{k_{1}\left(2^{-j(\xi)+1} \xi\right), \ldots, k_{n-1}\left(2^{-j(\xi)+1} \xi\right)\right\} ;
$$

if $D\left(2^{-j(\xi)} \xi\right)=0$, then

$$
\begin{aligned}
& k_{n}\left(2^{-j(\xi)-n(\xi)+1} \xi-2^{-n(\xi)}\right) \in K_{n-1}\left(2^{-j(\xi)-n(\xi)+1} \xi-2^{-n(\xi)}\right) \backslash \\
& \left\{k_{1}\left(2^{-j(\xi)-n(\xi)+1} \xi-2^{-n(\xi)}\right), \ldots, k_{n-1}\left(2^{-j(\xi)-n(\xi)+1} \xi-2^{-n(\xi)}\right)\right\} .
\end{aligned}
$$

Proof. First, Proposition 1 of [1] ensures the existence of $j(\xi)$ and $n(\xi)$.

Second, recall that the preceding lemma gives us an explicit formula for the function $k_{1}$ of Lemma 3.7 .

Let us repeat the inductive construction from the proof of Theorem 3.9 . Suppose that $k_{1}, \ldots, k_{n-1}$ have been constructed for some $n \geq 2$. Let $\xi \in T_{n}$. If $j(\xi) \geq 2$, then $D\left(2^{-j} \xi-1 / 2\right)=0$ for all $j=1, \ldots, j(\xi)-1$, so

$$
D\left(2^{-j} \xi\right)=D\left(2^{-j} \xi\right)+D\left(2^{-j} \xi-1 / 2\right)=D\left(2^{-(j-1)} \xi\right)+1
$$

for all $j=1, \ldots, j(\xi)-1$. Since $D(\xi) \geq n$, this implies

$$
D\left(2^{-j} \xi\right) \geq n, \quad j=0, \ldots, j(\xi)-1 .
$$

Moreover, from Remark 3.10 , it follows that $k_{n}(\xi)$ satisfies

$$
k_{n}(\xi)=2^{j(\xi)-1} k_{n}\left(2^{-(j(\xi)-1)} \xi\right) .
$$

Next, if $D\left(2^{-j(\xi)} \xi\right) \geq 1$, then we define $k_{n}\left(2^{-(j(\xi)-1)} \xi\right) \in K_{n-1}\left(2^{-j(\xi)+1} \xi\right) \backslash$ $\left\{k_{1}\left(2^{-j(\xi)+1} \xi\right), \ldots, k_{n-1}\left(2^{-j(\xi)+1} \xi\right)\right\}$ by using 3.9 .

It remains to consider the case $D\left(2^{-j(\xi)} \xi\right)=0$. Then $\xi \in[0,1 / 2)$ since $D$ is positive on $[-1 / 4,0)$. Therefore, $2^{-j(\xi)} \xi-1 / 2 \in[-1 / 2,0)$, so

$$
D\left(2^{-j}\left(2^{-j(\xi)} \xi-1 / 2\right)\right) \geq 1, \quad j \geq 0 .
$$

Using (3.14), we have

$D\left(2^{-j(\xi)} \xi-1 / 2\right)=D\left(2^{-j(\xi)} \xi\right)+D\left(2^{-j(\xi)} \xi-1 / 2\right)=D\left(2^{-(j(\xi)-1)} \xi\right)+1>n ;$ thus, $2^{-j(\xi)} \xi-1 / 2 \in T_{n}$. Furthermore, by the choice of $n(\xi)$,

$$
D\left(2^{-j}\left(2^{-j(\xi)} \xi-1 / 2\right)+1 / 2\right)=0, \quad j=0, \ldots, n(\xi)-1 .
$$

Hence,

$$
\begin{aligned}
D\left(2^{-j}\left(2^{-j(\xi)} \xi-1 / 2\right)\right) & \\
& =D\left(2^{-j}\left(2^{-j(\xi)} \xi-1 / 2\right)\right)+D\left(2^{-j}\left(2^{-j(\xi)} \xi-1 / 2\right)+1 / 2\right) \\
& =D\left(2^{-(j-1)}\left(2^{-j(\xi)} \xi-1 / 2\right)\right)+1
\end{aligned}
$$


for $j=0, \ldots, n(\xi)-1$. As $D\left(2^{-j(\xi)} \xi-1 / 2\right)>n$, this implies $D\left(2^{-j}\left(2^{-j(\xi)} \xi-\right.\right.$ $1 / 2))>n$ for all $j=0, \ldots, n(\xi)-1$. Hence, $2^{-(n(\xi)-1)}\left(2^{-j(\xi)} \xi-1 / 2\right) \in T_{n}$. Since $D\left(2^{-n(\xi)}\left(2^{-j(\xi)} \xi-1 / 2\right)+1 / 2\right) \geq 1$ and $D\left(2^{-n(\xi)}\left(2^{-j(\xi)} \xi-1 / 2\right)\right) \geq 1$ (which follows from (3.16)), we define

$$
\begin{aligned}
k_{n}\left(2^{-(n(\xi)-1)}\left(2^{-j(\xi)} \xi-1 / 2\right)\right) \in K_{n-1}\left(2^{-j(\xi)-n(\xi)+1} \xi-2^{-n(\xi)}\right) \backslash \\
\left\{k_{1}\left(2^{-j(\xi)-n(\xi)+1} \xi-2^{-n(\xi)}\right), \ldots, k_{n-1}\left(2^{-j(\xi)-n(\xi)+1} \xi-2^{-n(\xi)}\right)\right\}
\end{aligned}
$$

using (3.9). Now we claim that the value $k_{n}(\xi)$ can be expressed in terms of $k_{n}\left(2^{-j(\xi)-n(\xi)+1} \xi-2^{-n(\xi)}\right)$. To see this, first observe that 3.14 implies $2^{-(j(\xi)-1)} \xi \in T_{n}$ and recall that $D\left(2^{-j(\xi)} \xi\right)=0$. Thus, by Remark 3.10 .

$$
k_{n}\left(2^{-(j(\xi)-1)} \xi\right)=2 k_{n}\left(2^{-j(\xi)} \xi-1 / 2\right)-1 .
$$

Furthermore, by (3.17) and Remark 3.10.

$$
k_{n}\left(2^{-j(\xi)} \xi-1 / 2\right)=2^{n(\xi)-1} k_{n}\left(2^{-(n(\xi)-1)}\left(2^{-j(\xi)} \xi-1 / 2\right)\right) .
$$

Therefore, 3.15), 3.18) and 3.19) imply

$$
\begin{aligned}
k_{n}(\xi) & =2^{j(\xi)-1} k_{n}\left(2^{-(j(\xi)-1)} \xi\right)=2^{j(\xi)} k_{n}\left(2^{-j(\xi)} \xi-1 / 2\right)-2^{j(\xi)-1} \\
& =2^{j(\xi)+n(\xi)-1} k_{n}\left(2^{-j(\xi)-n(\xi)+1} \xi-2^{-n(\xi)}\right)-2^{j(\xi)-1} .
\end{aligned}
$$

We give a couple of examples to illustrate our technique for obtaining wavelets from dimension functions.

EXAmple 3.18. Consider the MRA dimension function $D(\xi)=1, \xi \in \mathbb{R}$. Clearly, we then have $\Delta=\mathbb{R}$ and $T_{n}=\emptyset$ for all $n \geq 2$. A direct application of Lemma 3.7 gives $k_{1} \equiv 0$; thus $S_{1}=T_{1}=[-1 / 2,1 / 2)$. Applying Theorem 3.5 , we end up with the Shannon wavelet set $W=[-1,-1 / 2) \cup[1 / 2,1)$.

EXAmple 3.19. Consider Journé's dimension function

$$
D(\xi)= \begin{cases}2, & \xi \in[-1 / 7,1 / 7)+\mathbb{Z}, \\ 1, & \xi \in([-1 / 2,-3 / 7) \cup[-2 / 7,-1 / 7) \cup[1 / 7,2 / 7) \cup[3 / 7,1 / 2))+\mathbb{Z}, \\ 0, & \text { otherwise. }\end{cases}
$$

Applying Lemma 3.7, we immediately get $k_{1} \equiv 0$; it follows that $S_{1}=T_{1}=$ $[-1 / 2,-3 / 7) \cup[-2 / 7,2 / 7) \cup[3 / 7,1 / 2)$.

Further, notice that $K_{1}(\xi)=\{-1,0\}$ or $K_{1}(\xi)=\{0,1\}$, for all $\xi \in T_{2}$, depending on $\operatorname{sgn} \xi$. Observe that, for $\xi \in T_{2}$, we have $D(\xi / 2) \geq 1$ and $D((\xi-\operatorname{sgn} \xi) / 2) \geq 1$, so we are in case (1) of the proof of Theorem 3.9. Hence, for $\xi \in T_{2}$, we must take $k_{2}(\xi) \in K_{1}(\xi) \backslash\left\{k_{1}(\xi)\right\}$. Since $k_{1}(\xi)=0$, this gives $k_{2}(\xi)=-1$ for $\xi \geq 0$ and $k_{2}(\xi)=1$ for $\xi<0$. Therefore, $S_{2}=$ $[-1,-6 / 7) \cup[6 / 7,1)$. Applying Theorem 3.5 , we obtain

$$
S=S_{1} \cup S_{2}=[-1,-6 / 7) \cup[-1 / 2,-3 / 7) \cup[-2 / 7,2 / 7) \cup[3 / 7,1 / 2) \cup[6 / 7,1)
$$


and

$$
\begin{gathered}
W=2 S \backslash S=[-2,-12 / 7) \cup[-4 / 7,-1 / 2) \cup[-3 / 7,-2 / 7) \\
\cup[2 / 7,3 / 7) \cup[1 / 2,4 / 7) \cup[12 / 7,2) .
\end{gathered}
$$

Recall that the original Journé wavelet set is given by

$$
W_{J}=[-16 / 7,-2) \cup[-1 / 2,-2 / 7) \cup[2 / 7,1 / 2) \cup[2,16 / 7) .
$$

Observe that $W$ has a smaller diameter than $W_{J}$. Moreover, we claim that the diameter of $W$ is smallest possible (as already mentioned in Remark 3.6(c)).

To prove this, let $k_{i}^{\prime}: T_{i} \rightarrow \mathbb{Z}, i=1,2$, be such that $S_{i}^{\prime}=\left\{\xi+k_{i}^{\prime}(\xi)\right.$ : $\left.\xi \in T_{i}\right\}, i=1,2$, satisfy the conditions of Remark 2.2. Let $S^{\prime}=S_{1}^{\prime} \cup S_{2}^{\prime}$ and $W^{\prime}=2 S^{\prime} \backslash S^{\prime}$.

For every $\xi \in T_{1}$ we have $k_{1}(\xi)=0$, so $\left|\xi+k_{1}(\xi)\right|=|\xi| \leq|\xi+k|$ for all $k \in \mathbb{Z}$ and therefore $\left|\xi+k_{1}(\xi)\right| \leq\left|\xi+k_{1}^{\prime}(\xi)\right|$. This means that the diameter of $S_{1}$ is smaller than that of $S_{1}^{\prime}$ (and of $S^{\prime}$ ). If $\xi \in T_{2}$ then $k_{2}(\xi)=$ $-\operatorname{sgn} \xi$, so $\left|\xi+k_{2}(\xi)\right|=|\xi-\operatorname{sgn} \xi| \leq|\xi+k|$ for all $k \in \mathbb{Z}, k \neq 0$. In particular, since $k_{1}^{\prime}(\xi) \neq k_{2}^{\prime}(\xi)$ and therefore $k_{1}^{\prime}(\xi) \neq 0$ or $k_{2}^{\prime}(\xi) \neq 0$, we have $\left|\xi+k_{2}(\xi)\right| \leq\left|\xi+k_{1}^{\prime}(\xi)\right|$ or $\left|\xi+k_{2}(\xi)\right| \leq\left|\xi+k_{2}^{\prime}(\xi)\right|$. Then $\left|\xi+k_{2}(\xi)\right| \leq$ $\max \left\{\left|\xi+k_{1}^{\prime}(\xi)\right|,\left|\xi+k_{2}^{\prime}(\xi)\right|\right\}$ for all $\xi \in T_{2}$, so $S_{2}$ has a smaller diameter than $S_{1}^{\prime} \cup S_{2}^{\prime}=S^{\prime}$. Therefore, $W$ has a smaller diameter than $W^{\prime}$.

ExAmple 3.20. Consider the unbounded dimension function $D$ constructed in $[1$. On $[-1 / 2,1 / 2)$, it is given by

$$
D(\xi)= \begin{cases}n, & \xi \in\left[-1 / 4^{n},-1 / 4^{n+1}\right) \cup\left[1 / 4^{n+1}, 1 / 4^{n}\right), n \in \mathbb{N}, \\ 0, & \xi \in F \cup H \cup(-F) \cup(-H), \\ 1, & \xi \in E \cup G \cup(-E) \cup(-G),\end{cases}
$$

where

$$
\begin{aligned}
& E=\bigcup_{k \geq 0} \bigcup_{n \geq 1} E_{k n}, \quad G=\bigcup \bigcup_{k \geq 1} G_{k n}, \\
& F=\bigcup_{k \geq 0} \bigcup_{n \geq 2} F_{k n}, \quad H=\bigcup \bigcup_{k \geq 1} H_{k \geq 2} \text {, }
\end{aligned}
$$

and

$$
\begin{aligned}
& E_{k n}=\left[-\frac{1}{3}-\frac{1}{3 \cdot 2^{2 k+1}}+\frac{1}{4^{k+n+1}},-\frac{1}{3}-\frac{1}{3 \cdot 2^{2 k+1}}+\frac{1}{2 \cdot 4^{k+n}}\right), \\
& F_{k n}=\left[-\frac{1}{3}-\frac{1}{3 \cdot 2^{2 k+1}}+\frac{1}{2 \cdot 4^{k+n}},-\frac{1}{3}-\frac{1}{3 \cdot 2^{2 k+1}}+\frac{1}{4^{k+n}}\right), \\
& G_{k n}=\left[-\frac{1}{3}+\frac{1}{3 \cdot 4^{k}}-\frac{1}{4^{k+n}},-\frac{1}{3}+\frac{1}{3 \cdot 4^{k}}-\frac{1}{2 \cdot 4^{k+n}}\right), \\
& H_{k n}=\left[-\frac{1}{3}+\frac{1}{3 \cdot 4^{k}}-\frac{2}{4^{k+n}},-\frac{1}{3}+\frac{1}{3 \cdot 4^{k}}-\frac{1}{4^{k+n}}\right) .
\end{aligned}
$$


We first observe that for $\xi \in T_{1}$ we have $2^{-j} \xi \in[-1 / 4,1 / 4), j \geq 0$; therefore $D\left(2^{-j} \xi\right) \geq 1, j \geq 0$. This gives $k_{1}(\xi)=0$ for $\xi \in T_{1}$ and $S_{1}=T_{1}$.

Notice that $T_{j}=\left[-1 / 4^{j}, 1 / 4^{j}\right), j \geq 2$. Since $D$ is symmetric we will work with $T_{j} \cap(0,1 / 2)$. Let $A_{n}=\left[1 / 4^{n+1}, 1 /\left(2 \cdot 4^{n}\right)\right)$ and $B_{n}=\left[1 /\left(2 \cdot 4^{n}\right), 1 / 4^{n}\right)$. Then $\bigcup_{n \geq j}\left(A_{n} \cup B_{n}\right)=\left(0,1 / 4^{j}\right)=T_{j} \cap(0,1 / 2)$.

It is easy to verify that for $\xi \in(0,1 / 16)$ we have

$$
j(\xi)= \begin{cases}1, & \xi \in \bigcup_{n \geq 2} B_{n}, \\ 2, & \xi \in \bigcup_{n \geq 2} A_{n},\end{cases}
$$

where $j(\xi)$ is as in Proposition 3.17 .

Let us determine $K_{j}(\xi)$ for $\xi \in\left(0,1 / 4^{j}\right), j \geq 2$. If $\xi \in \bigcup_{n \geq j} A_{n}$ then $D((\xi-1) / 2)=0$ and $D(\xi / 2)=D(\xi)+1 \geq j$, so $l_{j}(\xi)=j$ and $r_{j}(\xi)=0$. If $\xi \in \bigcup_{n \geq j} B_{n}$ then $D((\xi-1) / 2)=1$ and $D(\xi / 2)=D(\xi) \geq j$, so $l_{j}(\xi)=j$ and $r_{j}(\xi)=1$. This gives, for $j \geq 2$,

(3.22) $\quad K_{j}(\xi)$

$$
= \begin{cases}\left\{2 k_{1}(\xi / 2), 2 k_{2}(\xi / 2), \ldots, 2 k_{j}(\xi / 2)\right\}, & \xi \in \bigcup_{n \geq j} A_{n}, \\ \left\{2 k_{1}(\xi / 2), 2 k_{1}((\xi-1) / 2)-1,2 k_{2}(\xi / 2), \ldots, 2 k_{j}(\xi / 2)\right\}, & \xi \in \bigcup_{n \geq j} B_{n} .\end{cases}
$$

Since $D\left(2^{-j(\xi)} \xi\right) \geq 1$ for all $\xi \in T_{2}$, by Proposition 3.17 we have

$$
k_{j}(\xi)=2^{j(\xi)-1} k_{j}\left(2^{-j(\xi)+1} \xi\right), \quad j \geq 2 .
$$

It now follows from 3.21 that

$$
k_{j}(\xi)=2 k_{j}(\xi / 2), \quad \xi \in \bigcup_{n \geq j} A_{n},
$$

where $k_{j}(\xi / 2) \in K_{j-1}(\xi / 2) \backslash\left\{k_{1}(\xi / 2), \ldots, k_{j-1}(\xi / 2)\right\}$ is chosen by minimality principle; furthermore, $\xi / 2 \in \bigcup_{n \geq j} B_{n}$.

Also, (3.21) implies that $k_{j}(\xi) \in K_{j-1}(\xi) \backslash\left\{k_{1}(\xi), \ldots, k_{j-1}(\xi)\right\}$ for $\xi \in$ $\bigcup_{n \geq j} B_{n}$. In particular, for $j=2$ and $\xi \in \bigcup_{n \geq 2} B_{n}$ we get

$$
\begin{aligned}
k_{2}(\xi) \in K_{1}(\xi) \backslash & \left\{k_{1}(\xi)\right\} \\
& =\left\{2 k_{1}(\xi / 2), 2 k_{1}((\xi-1) / 2)-1\right\} \backslash\{0\}=\{-1\} .
\end{aligned}
$$

For $j=3$ and $\xi \in \bigcup_{n \geq 3} B_{n}$ we get, using 3.22 ,

$$
k_{3}(\xi) \in K_{2}(\xi) \backslash\left\{k_{1}(\xi), k_{2}(\xi)\right\}=\left\{0,-1,2 k_{2}(\xi / 2)\right\} \backslash\{0,-1\}=\left\{2 k_{2}(\xi / 2)\right\} .
$$

In general, for $j \geq 3$, we get

$$
k_{j}(\xi)=2 k_{j-1}(\xi / 2), \quad \xi \in \bigcup_{n \geq j} B_{n},
$$

and moreover $\xi / 2 \in \bigcup_{n \geq j} A_{n}$. Finally, using 3.23, 3.24 and 3.25, we 
conclude that

$$
k_{j}(\xi)= \begin{cases}-2^{2 j-3}, & \xi \in \bigcup_{n \geq j} A_{n}, \\ -2^{2 j-4}, & \xi \in \bigcup_{n \geq j} B_{n},\end{cases}
$$

for $j \geq 2$. Then, for $j \geq 2$,

$$
\begin{aligned}
S_{j}= & \bigcup_{n \geq j}\left(\left(A_{n}-2^{2 j-3}\right) \cup\left(B_{n}-2^{2 j-4}\right)\right) \\
& \cup \bigcup_{n \geq j}\left(\left(-A_{n}+2^{2 j-3}\right) \cup\left(-B_{n}+2^{2 j-4}\right)\right),
\end{aligned}
$$

and $W=2 S \backslash S=W_{+} \cup\left(-W_{+}\right)$where

$$
\begin{aligned}
W_{+}= & 2 E_{01} \cup \bigcup_{k \geq 1} \bigcup_{n \geq 1} 2 E_{k n} \cup \bigcup_{k \geq 0} \bigcup_{n \geq 2} F_{k n} \cup \bigcup_{k \geq 1} \bigcup_{n \geq 1} 2 G_{k n} \\
& \cup \bigcup_{k \geq 1} \bigcup_{n \geq 2} H_{k n} \cup \bigcup_{n \geq 2}\left(2 A_{n}-2^{2 n-2}\right) \cup \bigcup_{n \geq 2}\left(2 B_{n}-2^{2 n-3}\right) .
\end{aligned}
$$

Finally, let us briefly discuss possible generalizations/extensions of Theorem 3.1. One can ask if it is possible to characterize more general dimension functions in a similar way. (Again, for simplicity, we restrict ourselves to the dyadic case on the real line.)

Recall from [12] that an integrable $\mathbb{Z}$-periodic function $D: \mathbb{R} \rightarrow \mathbb{N} \cup\{0\}$ is a dimension function of some orthonormal wavelet if and only if $D$ satisfies (a), (b) and

( $\Delta) \sum_{k \in \mathbb{Z}} \chi_{\Delta}(\xi+k) \geq D(\xi)$ where $\Delta=\left\{\xi \in \mathbb{R}: D\left(2^{-j} \xi\right) \geq 1, \forall j \geq 0\right\}$.

Thus, conditions (c) and (d) from Theorem 3.1 are equivalent to $(\Delta)$, provided that (a) and (b) are satisfied.

It is well known that one can similarly characterize dimension functions of Parseval frame wavelets as well as dimension functions of GMRA's. For the reader's convenience we state the latter result from [9].

Theorem A ([9, Theorem 3.6]). Let $D: \mathbb{R} \rightarrow \mathbb{N} \cup\{0\}$ be a measurable $\mathbb{Z}$-periodic function. Then $D$ is the dimension function of the core space $V_{0}$ of some GMRA $\left(V_{j}\right)$ if and only if the following conditions are satisfied:

(a) $\liminf _{j \rightarrow \infty} D\left(2^{-j} \xi\right) \geq 1$ a.e.;

(b) $D(\xi)+D(\xi+1 / 2) \geq D(2 \xi)$ a.e.;

( $\Delta) \sum_{k \in \mathbb{Z}} \chi_{\Delta}(\xi+k) \geq D(\xi)$ a.e., where $\Delta=\left\{\xi \in \mathbb{R}: D\left(2^{-j} \xi\right) \geq 1\right.$, $\forall j \geq 0\}$.

So it is natural to ask if one can prove a variant of Theorem $\mathrm{A}$ in which $(\Delta)$ is replaced by (c) and (d). It turns out the answer is negative. As a counterexample, consider the function $D(\xi)=\chi_{\Omega}$ where $\Omega=S+\mathbb{Z}$ and $S=[-1 / 4,1 / 4)$. It is easy to verify that $D$ is the dimension function of the 
semi-orthogonal Parseval frame wavelet $\hat{\psi}=\chi_{W}$ with $W=2 S \backslash S$; hence $D$ is the dimension function of the core space of the underlying GMRA. Thus, $D$ satisfies the conditions of the above Theorem A. On the other hand, $D$ obviously does not satisfy

(c) for a.e. $\xi$ with $D(\xi) \geq 1$ there is $j \in \mathbb{N}$ satisfying $D\left(2^{-j} \xi+1 / 2\right) \geq 1$.

Quite surprisingly, it can be shown that an analogue of our Theorem 3.1 is valid if one modifies the above condition (c):

Theorem B. Let $D: \mathbb{R} \rightarrow \mathbb{N} \cup\{0\}$ be a measurable $\mathbb{Z}$-periodic function. Then $D$ is the dimension function of the core space $V_{0}$ of some GMRA $\left(V_{j}\right)$ if and only if the following conditions are satisfied:

(a) $\liminf _{j \rightarrow \infty} D\left(2^{-j} \xi\right) \geq 1$ a.e.;

(b) $D(\xi)+D(\xi+1 / 2) \geq D(2 \xi)$ a.e.;

$\left(\mathrm{c}^{\prime}\right)$ for a.e. $\xi$ with $D(\xi) \geq 2$ there is $j \in \mathbb{N}$ satisfying $D\left(2^{-j} \xi+1 / 2\right) \geq 1$;

(d) for a.e. $\xi$ with $D(\xi) \geq 1$ there is $k \in \mathbb{Z}$ such that $D\left(2^{-j}(\xi+k)\right) \geq 1$ for all $j \in \mathbb{Z}, j \geq 0$.

In particular, this implies that our Theorem 3.1 remains true if one replaces $(\mathrm{c})$ with $\left(\mathrm{c}^{\prime}\right)$. The details will appear elsewhere.

Acknowledgments. We thank the referee for several valuable suggestions that helped us to improve the presentation of our results.

\section{References}

[1] Lj. Arambašić, D. Bakić and R. Rajić, Dimension functions of orthonormal wavelets, J. Fourier Anal. Appl. 13 (2007), 331-356.

[2] N. Arcozzi, B. Behera, and S. Madan, Large classes of minimally supported frequency wavelets of $L^{2}(\mathbb{R})$ and $H^{2}(\mathbb{R})$, J. Geom. Anal. 13 (2003), 557-579.

[3] P. Auscher, Solution of two problems on wavelets, ibid. 5 (1995), 181-236.

[4] L. Baggett, J. Courter and K. Merrill, The construction of wavelets from generalized conjugate mirror filters in $L^{2}\left(\mathbb{R}^{n}\right)$, Appl. Comput. Harmon. Anal. 13 (2002), 201223.

[5] L. Baggett, P. Jorgensen, K. Merrill and J. Packer, Construction of Parseval wavelets from redundant filter systems, J. Math. Phys. 46 (2005), 083502.

[6] L. Baggett, H. Medina and K. Merrill, Generalized multiresolution analyses, and a construction procedure for all wavelet sets in $\mathbb{R}^{n}$, J. Fourier Anal. Appl. 5 (1999), 563-573.

[7] D. Bakić, Semi-orthogonal Parseval frame wavelets and generalized multiresolution analyses, Appl. Comput. Harmon. Anal. 21 (2006), 281-304.

[8] D. Bakić, On admissible generalized multiresolution analyses, Grazer Math. Berichte 348 (2006), 15-30.

[9] M. Bownik and Z. Rzeszotnik, The spectral function of shift invariant spaces, Michigan Math. J. 51 (2003), 387-414.

[10] - - - On the existence of multiresolution analysis for framelets, Math. Ann. 332 (2005), 705-720. 
[11] M. Bownik and Z. Rzeszotnik, Construction and reconstruction of tight framelets and wavelets via matrix mask functions, J. Funct. Anal. 332 (2009), 1065-1105.

[12] M. Bownik, Z. Rzeszotnik and D. Speegle, A characterization of dimension functions of wavelets, Appl. Comput. Harmon. Anal. 10 (2001), 71-92.

[13] X. Dai, D. R. Larson and D. M. Speegle, Wavelet sets in $\mathbb{R}^{n}$, J. Fourier Anal. Appl. 3 (1997), 451-456.

[14] X. Fang and X. Wang, Construction of minimally supported frequency wavelets, ibid. 2 (1996), 315-327.

[15] G. Gripenberg, A necessary and sufficient condition for the existence of a father wavelet, Studia Math. 114 (1995), 207-226.

[16] E. Hernández, X. Wang and G. Weiss, Smoothing minimally supported frequency wavelets I, J. Fourier Anal. Appl. 2 (1996), 329-340.

[17] E. Hernández and G. Weiss, A First Course on Wavelets, Stud. Adv. Math., CRC Press, 1996.

[18] K. Merrill, Simple wavelet sets for scalar dilations in $\mathbb{R}^{2}$, in: Representations, Wavelets and Frames, Appl. Numer. Harmon. Anal., Birkhäuser Boston, Boston, MA, 2008, 177-192.

[19] X. Wang, The study of wavelets from the properties of their Fourier transforms, Ph.D. Thesis, Washington Univ. in St. Louis, 1995.

Ljiljana Arambašić, Damir Bakić

Department of Mathematics

University of Zagreb

Bijenička c. 30

10000 Zagreb, Croatia

E-mail: arambas@math.hr bakic@math.hr
Rajna Rajić Faculty of Mining, Geology and Petroleum Engineering University of Zagreb Pierottijeva 6 10000 Zagreb, Croatia E-mail: rajna.rajic@zg.t-com.hr

Received April 30, 2009

Revised version November 20, 2009 